\title{
Mature neutrophils suppress T cell immunity in ovarian cancer microenvironment
}

Kelly L. Singel, ${ }^{1}$ Tiffany R. Emmons, ${ }^{1}$ ANM Nazmul H. Khan, ${ }^{2}$ Paul C. Mayor, ${ }^{3}$ Shichen Shen, ${ }^{4}$ Jerry T. Wong, ${ }^{5}$ Kayla Morrell, ${ }^{6}$ Kevin H. Eng, ${ }^{6}$ Jaron Mark, ${ }^{3}$ Richard B. Bankert, ${ }^{7}$ Junko Matsuzaki, ${ }^{8}$ Richard C. Koya, ${ }^{8}$ Anna M. Blom, ${ }^{9}$ Kenneth R. McLeish, ${ }^{10}$ Jun Qu, ${ }^{4}$ Sanjay Ram, ${ }^{11}$ Kirsten B. Moysich, ${ }^{12}$ Scott I. Abrams, ${ }^{1}$ Kunle Odunsi, ${ }^{3,8}$ Emese Zsiros, ${ }^{3,8}$ and Brahm H. Segal ${ }^{1,2,13}$

'Department of Immunology, ${ }^{2}$ Department of Internal Medicine, and ${ }^{3}$ Department of Surgery, Division of Gynecologic Oncology, Roswell Park Comprehensive Cancer Center, Buffalo, New York, USA. ${ }^{4}$ New York State Center of Excellence Bioinformatics and Life Sciences, University at Buffalo, Buffalo, New York, USA. ${ }^{5}$ Department of Pathology and Laboratory Medicine and ${ }^{6}$ Department of Biostatistics and Bioinformatics, Roswell Park Comprehensive Cancer Center, Buffalo, New York, USA. 'Department of Microbiology and Immunology, University at Buffalo Jacobs School of Medicine and Biomedical Sciences, Buffalo, New York, USA. ${ }^{8}$ Center for Immunotherapy, Roswell Park Comprehensive Cancer Center, Buffalo, New York, USA. ${ }^{9}$ Division of Medical Protein Chemistry, Department of Translational Medicine, Lund University, Malmö, Sweden. ${ }^{10}$ Department of Medicine, University of Louisville School of Medicine, Louisville, Kentucky, USA. "Department of Medicine, University of Massachusetts Medical School, Worcester, Massachusetts, USA. ${ }^{12}$ Department of Cancer Prevention and Control, Roswell Park Comprehensive Cancer Center, Buffalo, New York, USA. ${ }^{13}$ Department of Medicine, Jacobs School of Medicine and Biomedical Sciences, University at Buffalo, Buffalo, New York, USA.

Epithelial ovarian cancer (EOC) often presents with metastases and ascites. Granulocytic myeloidderived suppressor cells are an immature population that impairs antitumor immunity. Since suppressive granulocytes in the ascites of patients with newly diagnosed EOC were morphologically mature, we hypothesized that PMN were rendered suppressive in the tumor microenvironment (TME). Circulating PMN from patients were not suppressive but acquired a suppressor phenotype (defined as $\geq 1 \log _{10}$ reduction of anti-CD3/CD28-stimulated T cell proliferation) after ascites supernatant exposure. Ascites supernatants (20 of 31 supernatants) recapitulated the suppressor phenotype in PMN from healthy donors. T cell proliferation was restored with ascites removal and restimulation. PMN suppressors also inhibited T cell activation and cytokine production. PMN suppressors completely suppressed proliferation in naive, central memory, and effector memory $T$ cells and in engineered tumor antigen-specific cytotoxic T lymphocytes, while antigen-specific cell lysis was unaffected. Inhibition of complement $\mathrm{C} 3$ activation and PMN effector functions, including CR3 signaling, protein synthesis, and vesicular trafficking, abrogated the PMN suppressor phenotype. Moreover, malignant effusions from patients with various metastatic cancers also induced the $[3$-dependent PMN suppressor phenotype. These results point to PMN impairing $\mathrm{T}$ cell expansion and activation in the TME and the potential for complement inhibition to abrogate this barrier to antitumor immunity.

Conflict of interest: KLS and BHS are coinventors on a provisional patent application based on this manuscript.

License: Copyright 2019, American Society for Clinical Investigation.

Submitted: May 21, 2018

Accepted: January 25, 2019

Published: March 7, 2019

\section{Reference information:} JCl Insight. 2019;4(5):e122311. https:// doi.org/10.1172/jii.insight.122311.

\section{Introduction}

Epithelial ovarian cancer (EOC) is typically diagnosed at advanced stages, presenting with peritoneal metastases and ascites accumulation. The tumor microenvironment (TME) of EOC is composed of immunological niches that influence tumor progression and response to therapy (1). There is growing recognition for ascites as a distinct part of the EOC TME that facilitates seeding of serosal surfaces, mediates resistance to chemotherapy, and impairs antitumor immunity (2). Ascites contains specific tumor-associated lymphocyte populations that are being explored for cellular therapy (3), as well as immunosuppressive myeloid cells $(4,5)$ and exosomes $(6)$ that are obstacles to antitumor immunity. Additional studies demonstrated distinct proteomic, glycosylation, and metabolic profiles in ascites that can affect tumor cell biology (7-9). 
The presence and volume of ascites at diagnosis of advanced EOC were associated with worse progression-free survival (PFS) and overall survival (OS) $(10,11)$. These findings point to both cellular and soluble constituents of ascites influencing metastasis, antitumor immune responses, and prognosis.

The critical role of $\mathrm{T}$ cell immunity in EOC was demonstrated by tumor-infiltrating $\mathrm{CD}^{+} \mathrm{T}$ cells predicting better outcomes $(12,13)$, while increased accumulation of Tregs was associated with worse outcomes (14). CD8 $8^{+} \mathrm{T}$ cells that recognized NY-ESO-1, a tumor antigen, had impaired effector functions, but inhibiting LAG-3 and PD-1 signaling augmented proliferation and cytokine production (15). However, anti-PD-1 and anti-PD-L1 inhibitors have been largely ineffective in patients with relapsed/refractory EOC, with overall responses of $9 \%-15 \%$ (16-18), raising the notion of other suppressive pathways in the TME as obstacles to immunotherapy.

Myeloid-derived suppressor cells (MDSC) are defined as immature myeloid cells that suppress $\mathrm{T}$ cell responses. They include myeloid progenitors and immature myeloid cells (19). Because MDSC markers overlap with other cell populations, phenotyping combined with demonstration of $\mathrm{T}$ cell suppression is optimal for identification of MDSC (20). Advanced cancer is associated with a myeloid bias characterized by increased frequencies of circulating granulocyte-monocyte progenitors that are skewed toward differentiating into granulocytes (21). Tumor-derived factors, such as G-CSF, GM-CSF, and IL-6, drive this myeloid bias (22) and result in a circulating and tumor-infiltrating MDSC population that accelerates tumor progression by suppressing $\mathrm{T}$ cell responses and releasing factors that promote metastasis. Cui et al. (23) found that MDSC in EOC triggered acquisition of stem cell-like features in cancer cells and increased metastatic potential, and they found that myeloid cell $\left(\mathrm{CD} 33^{+}\right)$accumulation was associated with worse outcomes. Tumor-associated PMN (TAN) can be broadly divided into N1 (antitumorigenic) or N2 (suppressive and protumorigenic) populations, with distinct transcriptional profiles and functional properties (24). These findings point to suppressive myeloid cells in the TME of EOC as barriers to antitumor immunity.

The concept of a myeloid bias can also apply to mature PMN. In patients with EOC, the pretreatment circulating PMN count (25) and the PMN/lymphocyte ratio (26) correlated with poor outcomes. Moreover, activated PMN can acquire a suppressive phenotype (27-29). There exists a gap in knowledge regarding the role of mature PMN as suppressor cells in the TME, as well as mechanisms for where and how PMN acquire a suppressive phenotype. The distinction between mature and immature suppressive granulocytes is mechanistically and therapeutically important. If circulating PMN are mature and acquire a suppressor phenotype within the TME, then therapeutic approaches should aim to disable their recruitment to the TME and target pathways driving suppression rather than aiming to modulate myeloid programming in the marrow.

Since suppressive granulocytes in the ascites of patients with newly diagnosed EOC were morphologically mature, we hypothesized that mature, circulating PMN acquired a suppressor phenotype after recruitment to the TME. We recently observed that circulating PMN from healthy donors acquired a suppressor phenotype after exposure to ascites supernatants (ASC) from patients with newly diagnosed advanced EOC (30). In the current study, we delineated mechanisms for this newly identified PMN suppressor phenotype. Inhibition of complement $\mathrm{C} 3$ activation and PMN effector functions, including CR3 signaling, protein synthesis, and vesicular trafficking, abrogated the PMN suppressor phenotype. Together, these results point to mature $\mathrm{PMN}$ as the immunosuppressive granulocyte population in the EOC microenvironment and identify complement activation and other pathways for therapeutic modulation.

\section{Results}

Ovarian cancer ascites induces patient circulating PMN to become T cell suppressive. Ascites from patients with newly diagnosed EOC contains monocytes/macrophages and granulocytes with variable immunosuppressive phenotypes $(4,5)$. Since MDSC are defined as immature, we compared the major populations of circulating and ascites WBC and their maturity based on standard cytologic criteria. In routine preoperative complete blood cell count $(\mathrm{CBC})$ testing, patients with newly diagnosed EOC had normal circulating WBC numbers and differentials. Granulocytes were $>99 \%$ mature segmented PMN, bands were $<1 \%$, and no immature granulocytes were observed (Table 1). We found no difference in the proportions of circulating $\mathrm{PMN}\left(\mathrm{CD} 45^{+} \mathrm{CD} 11 \mathrm{~b}^{+} \mathrm{CD} 33^{\text {mid }} \mathrm{CD} 15^{+} \mathrm{CD} 14^{-} \mathrm{DR}{ }^{-}\right)$and monocytes $\left(\mathrm{CD} 45^{+} \mathrm{CD} 11 \mathrm{~b}^{+} \mathrm{C}-\right.$ $\mathrm{D} 33^{\text {hi }} \mathrm{CD} 15^{-} \mathrm{CD} 14^{+} \mathrm{DR}^{+}$) between patients with high-grade serous ovarian cancer (HGSOC; accounts for majority of all cases) and female patients undergoing surgery for a benign adnexal mass (control blood) (Figure 1A and Supplemental Figure 1; supplemental material available online with this article; https:// doi.org/10.1172/jci.insight.122311DS1). We analyzed the cellular composition and morphology of gran- 
ulocytes in Wright Giemsa-stained cytospins of ascites from patients with newly diagnosed metastatic HGSOC (Figure 1, B and C). The granulocytes had segmented nuclei with prominent filaments characteristic of mature PMN. No immature granulocytes were observed. The ascites PMN/lymphocyte ratio was 1.03 (95\% CI, 0.21-1.8, SEM 0.4) (Figure 1D). These results demonstrate that the inflammatory microenvironment in ascites is distinct from blood, and circulating and ascites PMN are morphologically mature.

Because we previously observed that ascites granulocytes suppressed stimulated T cell proliferation (5), we evaluated whether circulating PMN from patients with advanced EOC were suppressive. We assessed the proliferation of anti-CD3/CD28-stimulated T cells from patients with newly diagnosed EOC $(n=4)$ after incubation with media, autologous PMN, and/or ASC. The coculture PMN/lymphocyte ratio was $1: 1$, corresponding to the mean ratio observed in ascites. Addition of either PMN or ascites alone resulted in negligible reductions in stimulated $\mathrm{T}$ cell proliferation (Figure 1, E and F). However, when added together, the interaction effect of PMN and ascites reduced T cell proliferation by a factor of $2.08 \log _{10}(95 \% \mathrm{CI}$, $1.26-2.90, P=0.0002$ ) (Figure $1 \mathrm{~F}$ ). These results establish that ascites induces mature PMN to acquire a suppressor phenotype and are consistent with the hypothesis that mature, circulating PMN acquire this suppressor phenotype upon recruitment to the TME.

Ovarian cancer ascites induces circulating PMN from healthy donors to acquire the suppressor phenotype. In patients with metastatic EOC, it is possible that tumor-derived factors could influence marrow and circulating granulocytes to render them more sensitive to the effects of ascites. We recently showed that ascites from patients with HGSOC induced the suppressor phenotype in PMN from healthy donors (30). In the current study, we extended these results to include a larger number of EOC ascites and histology other than HGSOC ( $n=31$; Table 2). PMN and T cells from a cohort of healthy donors were used for each experiment. Similar to patient PMN, ascites rendered PMN suppressive when cocultured with autologous $\mathrm{T}$ cells stimulated with anti-CD3/CD28 microbeads and soluble anti-CD3/CD28 Ab (Figure 2A). Again, addition of PMN or ascites alone resulted in small biological effects $\left(0.21\right.$ and $0.24 \log _{10}$ reductions).

We stratified ascites $(n=31)$ into 3 categories based on the induction of a PMN suppressor phenotype, where $\mathrm{X}$ equals a reduction in proliferation as compared with anti-CD3/CD28-stimulated $\mathrm{T}$ cells alone: suppressors $\left(X \geq 1 \log _{10}\right)$, intermediate suppressors $\left(0.5 \log _{10} \leq \mathrm{X}<1 \log _{10}\right)$, and nonsuppressors $(\mathrm{X}<0.5$ $\log _{10}$ ) (Figure 2B and Table 2). These results include ascites from $n=22$ HGSOC patients reported in our recent study (30). From this point on, we preselected ascites known to induce the PMN suppressor phenotype $\left(X \geq 1 \log _{10}\right)$ in order to evaluate mechanisms for PMN-mediated suppression. Together, these findings show that mature PMN are the suppressive granulocytic population in EOC ascites and are rendered suppressive by factors in the TME.

An effective antitumor response requires expansion and activation of tumor antigen-specific effector $\mathrm{T}$ cells in the TME. To determine whether the PMN suppressor phenotype affected central and effector memory $\mathrm{T}$ cells, we isolated naive $\left(\mathrm{CD}^{+} \mathrm{CD} 45 \mathrm{RA}^{+} \mathrm{RO}^{-} \mathrm{CD} 62 \mathrm{~L}^{+}\right)$, central memory $\left(\mathrm{CD}^{+} \mathrm{CD}^{-} 5 \mathrm{RA}^{-} \mathrm{RO}^{+} \mathrm{C}-\right.$ $\left.\mathrm{D}_{62} \mathrm{~L}^{+}\right)$, and effector memory $\left(\mathrm{CD}^{+} \mathrm{CD} 45 \mathrm{RA}^{-} \mathrm{RO}^{+} \mathrm{CD}^{2} 2 \mathrm{~L}^{-}\right) \mathrm{T}$ cell populations from blood (Supplemental Figure 2). All anti-CD3/CD28-stimulated T cell populations were suppressed by the PMN suppressor phenotype (Figure 2, C-E). Since the PMN suppressor phenotype had a similar effect on naive, central memory, and effector memory $\mathrm{T}$ cells, we used unfractionated $\mathrm{T}$ cells in subsequent experiments. These findings showing that the PMN suppressor phenotype acts on the major circulating $\mathrm{T}$ cell populations, including effector memory $\mathrm{T}$ cells that drive antitumor immunity, support the importance of suppressive $\mathrm{PMN}$ as obstacles to antitumor immunity.

Suppressed $T$ cells are viable, and suppression is reversible. We asked whether the observed reduction in stimulated $\mathrm{T}$ cell proliferation when cocultured with PMN and ascites was due to T cell apoptosis. The proportion of apoptotic stimulated $\mathrm{T}$ cells cocultured with media, ASC, and/or PMN ranged from $17 \%-27 \%$ (Figure $2 \mathrm{~F}$ ). In addition, when $\mathrm{T}$ cells were cocultured with ascites and PMN, T cell proliferation was restored with ascites removal and anti-CD3/CD28 restimulation (Figure 2, G and $\mathrm{H}$ ). Addition of recombinant IL-2 (rIL-2) to cocultures at 48 hours did not reverse T cell suppression (Figure 2I). These results argue against $\mathrm{T}$ cell apoptosis as a mechanism for the PMN suppressor phenotype and show the potential for reversibility of $\mathrm{T}$ cell suppression.

Next, we carried out a series of experiments to identify the time frame of $\mathrm{T}$ cell suppression in relation to anti-CD3/CD28 stimulation and exposure to PMN and ascites. When T cells were anti-CD3/CD28-stimulated for 18 hours (Supplemental Figure 3A) or 1-6 hours (Supplemental Figure 3B) and then cocultured with ascites and PMN, T cell proliferation was unimpaired. However, when T cells were cocultured with 
Table 1. Patients with newly diagnosed EOC have normal circulating WBC numbers and differentials

\begin{tabular}{|c|c|c|c|c|}
\hline & \multicolumn{4}{|c|}{ Suppressor status } \\
\hline & $\begin{array}{l}\text { Suppressor } \\
\left(X \geq 1 \log _{10}\right)\end{array}$ & $\begin{array}{c}\text { Intermediate } \\
\left(0.5 \log _{10} \leq X<1 \log _{10}\right)\end{array}$ & $\begin{array}{c}\text { Non } \\
\left(X<0.5 \log _{10}\right)\end{array}$ & $P$ value \\
\hline$n=31$ & 20 & 8 & 3 & \\
\hline Preoperative CBC & $16 / 20$ & $7 / 8$ & $3 / 3$ & \\
\hline WBC $\left(1 \times 10^{9}\right.$ cells $\left./ I\right)$ & 8.6 & 8.5 & 9.3 & 0.94 \\
\hline$\% \mathrm{HCT}$ & 36.1 & 35.6 & 39.6 & 0.62 \\
\hline PLT $\left(1 \times 10^{9}\right.$ cells $\left./ \mathrm{I}\right)$ & 411.0 & 565.0 & 352.3 & 0.22 \\
\hline Differential & $13 / 20$ & $6 / 8$ & $3 / 3$ & \\
\hline PMN $\left(1 \times 10^{9}\right.$ cells $\left./ \mathrm{I}\right)$ & 6.3 & 6.5 & 6.1 & 0.93 \\
\hline $\begin{array}{l}\text { Lymphocyte } \\
\left(1 \times 10^{9} \text { cells/I) }\right.\end{array}$ & 1.3 & 1.2 & 2.2 & 0.56 \\
\hline Monocyte $\left(1 \times 10^{9}\right.$ cells/I) & 0.6 & 0.5 & 0.7 & 0.71 \\
\hline NLR & 4.7 & 5.5 & 2.8 & 0.73 \\
\hline Manual counts & $7 / 20$ & $3 / 8$ & $1 / 3$ & \\
\hline \% Segmented PMN & 67.0 & 77.5 & 73.2 & 0.30 \\
\hline$\%$ Bands & 1.0 & NR & NR & \\
\hline \% Metamyelocytes & NR & NR & NR & \\
\hline
\end{tabular}

Data are based on review of electronic health records at Roswell Park Comprehensive Cancer Center. Statistical comparisons were by ANOVA with Tukey post hoc test. CBC, complete blood cell count; WBC, white blood cell; HCT, hematocrit; PLT, platelet; NLR, neutrophil/lymphocyte ratio; NR, none recorded.

ascites and PMN, followed by addition of anti-CD3/CD28 stimulation at various time points, suppression of $\mathrm{T}$ cell proliferation occurred when anti-CD3/CD28 was added within 1 hour of coculture but was lost at 2 hours (Supplemental Figure 3C). Surface expression of CD3 and CD28 on T cells after incubation with ascites and/or PMN was similar to T cells incubated with media (Supplemental Figure 3, D and E), indicating that the mechanism for $\mathrm{T}$ cell suppression is not due to loss of CD3 and CD28. These results show that the PMN suppressor phenotype requires PMN and ascites exposure early after T cell stimulation, is reversible, and raises the potential for therapeutically abrogating the suppressor phenotype.

$P M N$ suppressor phenotype requires $T$ cell contact and complement $C 3$ activation. We previously observed that ascites stimulated PMN degranulation and the generation of PMN extracellular traps (NETs) (30), raising the possibility that soluble products may be released into the coculture and mediate suppression of $\mathrm{T}$ cells. In the current study, when we exposed PMN to ascites for 6 hours and then added the PMN pellets or supernatants to anti-CD3/CD28-stimulated T cells, proliferation was unimpaired (Supplemental Figure $3 \mathrm{~F})$. In addition, separation of PMN and T cells using a transwell system resulted in abrogation of suppression (Figure 3A), suggesting that cell contact between PMN and T cells is required for suppression.

Complement receptor 3 (CR3; Mac-1; CD11b/CD18) mediates a critical step in PMN recruitment and cell-to-cell adhesion by binding to ICAM-1 on endothelial and T cells. Pretreating PMN with anti-CD11b $\mathrm{Ab}$ abrogated suppression, while pretreating T cells with anti-ICAM-1 Ab had no effect (Figure 3B). Pretreating PMN or T cells with IgG1 as an isotype control had no effect. In humans, endotoxin (LPS) challenge or severe injury resulted in a subset of circulating PMN (CD11 ${ }^{\text {bright }} C D 62 L^{\text {dim }} C D 11 b^{\text {bright }} C D 16^{\text {bright }}$ ) that mediated $\mathrm{T}$ cell suppression through oxidant generation and CD11b (31). We observed that, after 24 hours, PMN in media or ascites variably downregulated CD62L and CD16 expression, but there was no discernible population with increased CD16 expression as compared with baseline (data not shown), suggesting that the PMN suppressor phenotype induced by ascites is distinct from circulating PMN suppressors induced by acute systemic inflammation. CR3 also binds $\mathrm{iC} 3 \mathrm{~b}$, a cleavage product of C3 that acts as an opsonin and mediates intracellular signaling. Pretreating PMN with either $\mathrm{C} 3 \mathrm{~b}$ or iC $3 \mathrm{~b}$ prior to coculture resulted in abrogation of the suppressor phenotype, suggesting a desensitizing effect on PMN (Figure 3C).

To determine if the factor in ascites inducing the PMN suppressor phenotype was complement related, we first evaluated if it was a heat-labile protein via heat inactivation (HI-ASC; Figure 3D) and proteinase-K digestion (PK-ASC; Supplemental Figure 4A) of ASC prior to addition to cocultures. Both treatments completely abrogated $\mathrm{T}$ cell suppression. Since ascites exosomes can inhibit $\mathrm{T}$ cell responses (6), we determined whether the suppressive factor was membrane associated or soluble. The ascites were ultra- 

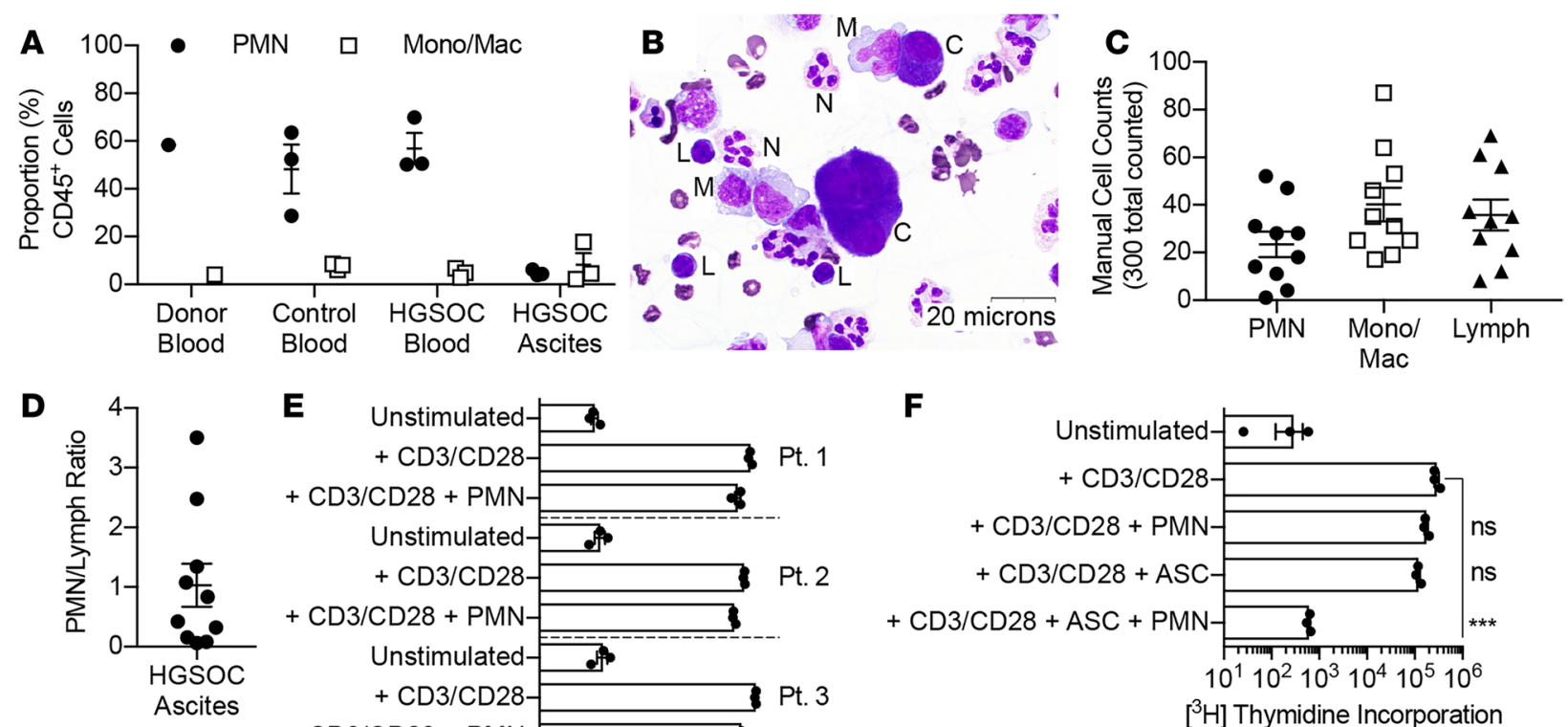

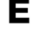

E Unstimulated-

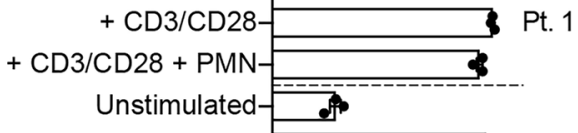

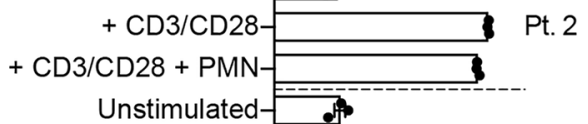

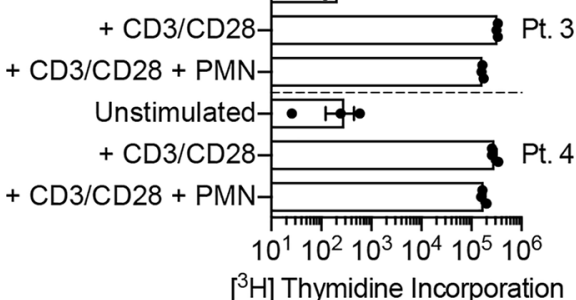

(Net CPM)
$\mathbf{F}$

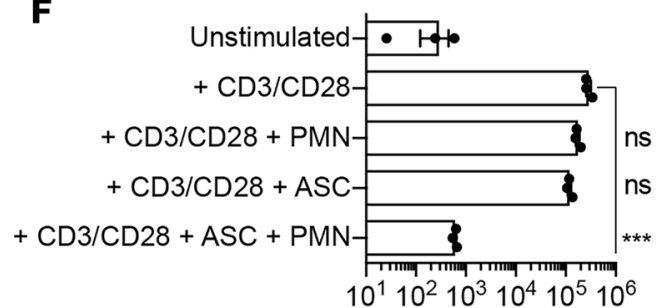

$\left[{ }^{3} \mathrm{H}\right]$ Thymidine Incorporation (Net CPM)

Figure 1. Ovarian cancer ascites induces circulating patient PMN to become suppressive. (A) The proportion of circulating WBC populations in a healthy donor $(n=1)$, control female patients undergoing surgery for a benign peritoneal mass $(n=3)$, and patients undergoing surgery for newly diagnosed HCSOC $(n=3)$ are similar but differ markedly from WBC populations in paired HCSOC ascites $(n=3)$. (B-D) Cytologic analysis of Wright Giemsa-stained cytospins of ascites from newly diagnosed HGSOC $(n=10)$. (B) Representative image showing mature PMN (N), monocytes/macrophages (M), lymphocytes $(\mathrm{L})$, and tumor cells (C). All PMN were morphologically mature with characteristic segmented nuclei. (C) WBC proportions were quantified. PMN, 4\%-52\%; monocytes/macrophage, 17\%-87\%; and lymphocytes, 8\%-69\%. (D) Mean PMN/lymphocyte ratio was 1.03 (95\% CI, 0.21-1.8, SEM 0.4). (E and F) T cells (CD3+) and PMN were isolated from patient blood and used in autologous coculture at 1:1 based on data in $\mathbf{D}(n=4)$. PMN and/or ascites supernatants (ASC; $50 \%$ final well volume) were added to anti-CD3/CD28-stimulated T cells. After 72 hours of coculture, $T$ cell proliferation was measured by [ ${ }^{3} \mathrm{H}$ ] thymidine incorporation (16-18 hours). (E) HGSOC patient circulating PMN were negligibly T cell suppressive. (F) ASC are not suppressive alone but induce patient PMN to suppress stimulated T cell proliferation by a factor of $2.08 \log _{10}(95 \% \mathrm{Cl}, 1.26-2.90)$. Symbols represent individual samples $(n)$, and bars represent $\pm \mathrm{SEM}$. Statistical comparisons were by ANOVA with Tukey post hoc test $\left({ }^{* *} P<0.001\right)$.

centrifuged to separate the membrane-rich (MR-ASC) and membrane-deplete (MD-ASC) fractions. The MR-ASC neither suppressed T cell proliferation alone nor in combination with PMN, while the MD-ASC rendered PMN suppressive (Supplemental Figure 4B). These results show that soluble, heat-labile proteins in ascites are required for the PMN suppressor phenotype.

C3 plays a central role in the activation of the 3 complement pathways: classical, alternative, and lectin. Compstatins are a family of peptides that inhibit complement activation by binding to native $\mathrm{C} 3$ and interfering with convertase formation and C3 cleavage, and they are being developed as therapeutics for complement-driven disorders $(32,33)$. To test the role of $\mathrm{C} 3$ activation in the PMN suppressor phenotype, we treated ascites with compstatin (CS-ASC; $250 \mu \mathrm{M} ; n=27$ ) (Figure 3, E-G) and Cp40 (Cp40-ASC; $20 \mu \mathrm{M} ; n=10$ ) (Figure $3 \mathrm{H}$ ) prior to coculture with PMN and T cells. Both completely abrogated the PMN suppressor phenotype, while scramble peptide (SCR-ASC) had no effect. The mean PMN viability (based on $\mathrm{PI}^{-}$annexin- $\mathrm{V}^{-}$) after 24-hour exposure to Cp40-ASC $(n=4)$ was $68 \% \pm 8 \%$, which was similar to untreated ascites $(80 \% \pm 8 \%)$ and SCR-ASC $(80 \% \pm 10 \%)$ (data not shown). PMN viability ranged between $41 \%-47 \%$ at 54 hours under these conditions; these results do not support excess PMN death as a mechanism for $\mathrm{Cp} 40$ abrogating the PMN suppressor phenotype. A concentration-titration study showed that $5 \mu \mathrm{M}$ Cp40 was sufficient to fully abrogate the PMN suppressor phenotype (Figure 3I).

To evaluate the role of downstream complement proteins in mediating the PMN suppressor phenotype, ascites were pretreated with $\mathrm{Ab}$ against $\mathrm{C} 5$ or with $\mathrm{OmCI}$, a peptide $\mathrm{C} 5$ inhibitor derived from the saliva of 
Table 2. Ascites stratification strategy based on the induction of a suppressor phenotype in PMN

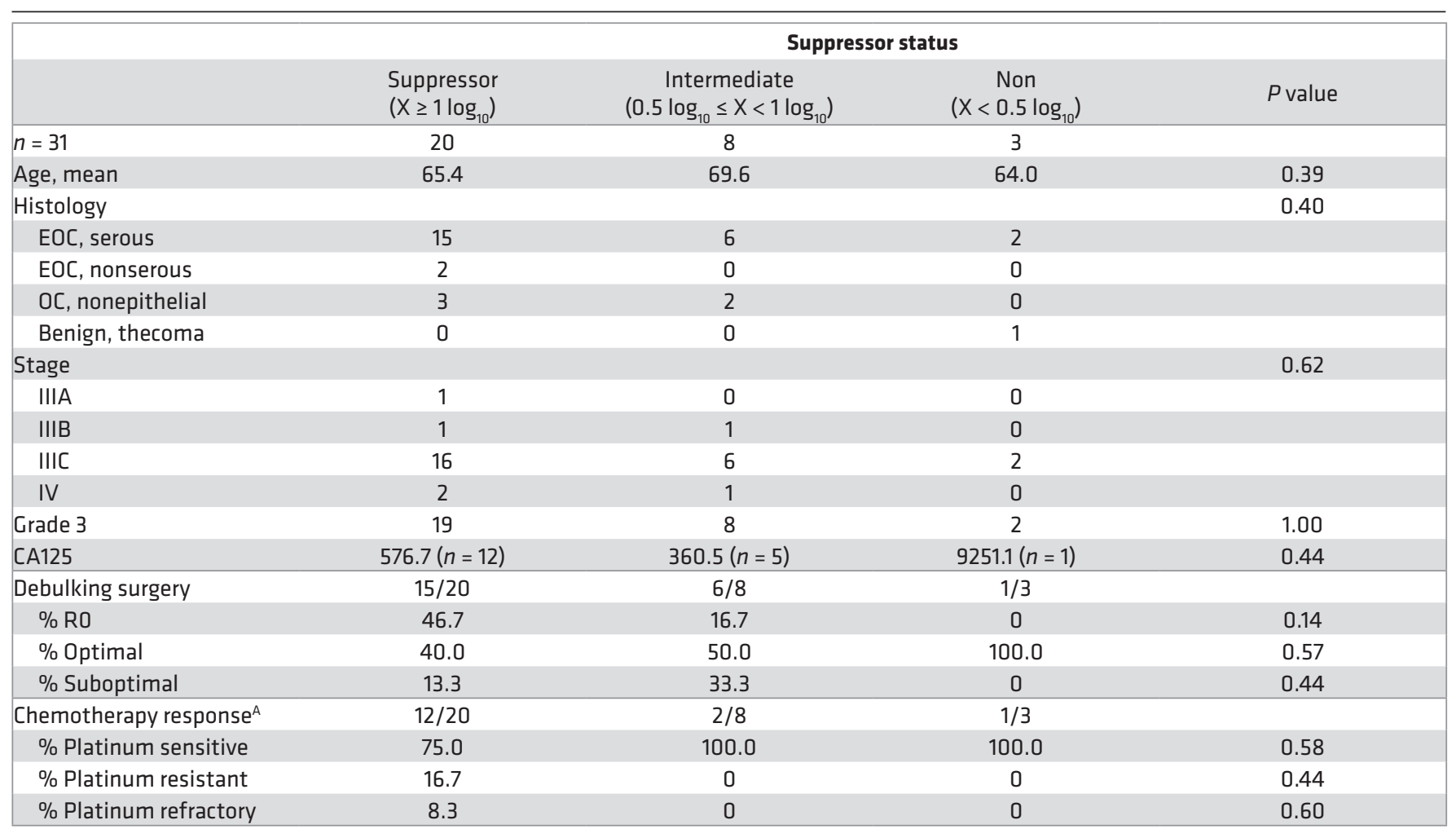

${ }^{A}$ RECiST Evaluation complete response after end of adjuvant chemotherapy. Ascites were stratified into 3 categories based on the induction of a suppressor phenotype in PMN, where $X$ equals a reduction in proliferation as compared with anti-CD3/CD28-stimulated T cells alone: suppressors ( $X \geq 1$ log 10 ), intermediate suppressors $\left(0.5 \log _{10} \leq X<1 \log _{10}\right)$, and nonsuppressors $\left(X<0.5 \log _{10}\right)$. Statistical comparisons were by ANOVA with Tukey post hoc test.

Ornithodoros moubata $(34,35)$, prior to coculture. Inhibiting C5, with either antibody or peptide, had a partial abrogating effect on $\mathrm{T}$ cell suppression, as compared with $\mathrm{Cp} 40$ that fully abrogated the PMN suppressor phenotype (Figure 3J). The membrane attack complex (MAC; C5b-C9) disrupts membranes of target cells, leading to cell lysis. Ab against $\mathrm{C}$, a required component of MAC, had no effect on $\mathrm{T}$ cell suppression. These results show that functional $\mathrm{CR} 3$ and activation of $\mathrm{C} 3$ are required, $\mathrm{C} 5$ has an intermediate effect, and MAC is unlikely to be involved in the PMN suppressor phenotype.

Finally, we asked whether the PMN suppressor phenotype induced by EOC ascites would also occur following PMN exposure to other malignant effusions. We observed a similar PMN suppressor phenotype when PMN and anti-CD3/CD28-stimulated T cells were cocultured with malignant pleural and ASC from patients with a number of metastatic cancers (18 of 20 samples induced a PMN suppressor or intermediate suppressor phenotype). Using samples that met the suppressor definition (Table 3), the PMN suppressor phenotype induced by malignant pleural and ascites was also abrogated by $\mathrm{Cp} 40$ treatment (Figure $3 \mathrm{~K}$ ). These data demonstrate the generalizability of our findings regarding the C3-dependent induction of the PMN suppressor phenotype in malignant effusions.

Ascites activates multiple PMN effector pathways that mediate suppression. We undertook a comprehensive analysis of the role of effector pathways in mediating the PMN suppressor phenotype. Since PMN degranulation can result in the release of suppressive products, including arginase-1 (28), we evaluated PMN surface expression for markers of fusion of primary (CD63), secondary and tertiary (CD66b) granules, and secretory vesicles (CD35; CR1) after 30- and 60-minute exposure to media, N-Formylmethionine-leucyl-phenylalanine (fMLF; positive control), ASC, or HI-ASC. CD63 surface expression was unaffected by ascites (Figure 4, A and B), and CD66b surface expression was no different between untreated ascites and HI-ASC (Figure 4, C and D). However, CD35/CR1 surface expression increased with ascites as compared with media and decreased again with HI-ASC (Figure 4, E and F). These results suggest that ascites induces variable effects on fusion of PMN granules and secretory vesicles. 


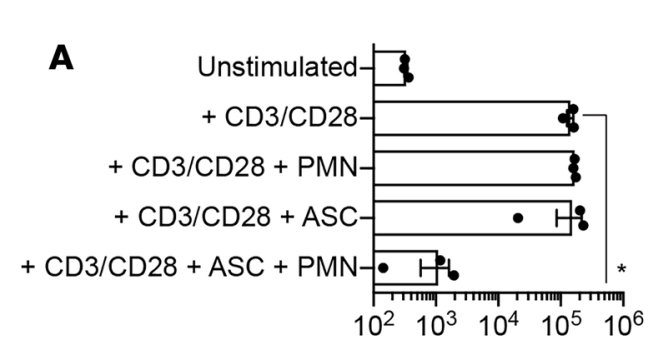

$\left[{ }^{3} \mathrm{H}\right]$ Thymidine Incorporation (Net CPM)

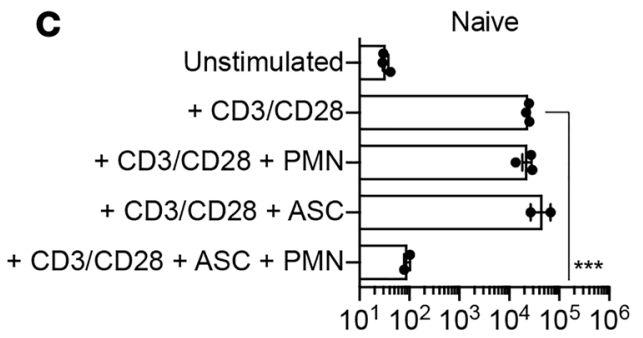

$\left[{ }^{3} \mathrm{H}\right]$ Thymidine Incorporation (Net CPM)

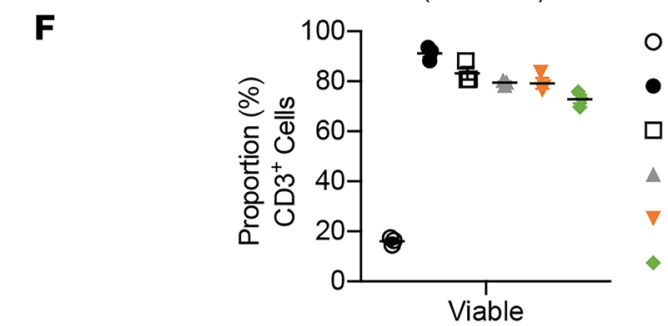

(Annexin $\mathrm{V}^{\text {neg }}$ )

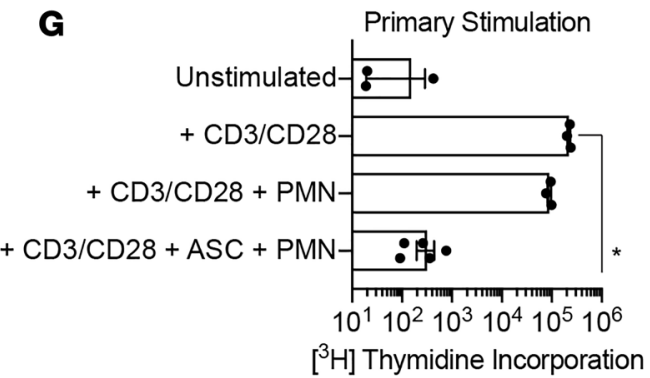

(Net CPM)

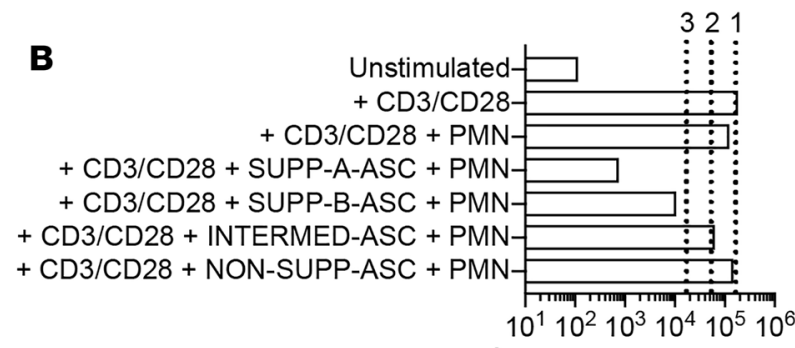

$\left[{ }^{3} \mathrm{H}\right]$ Thymidine Incorporation (Net CPM)

D

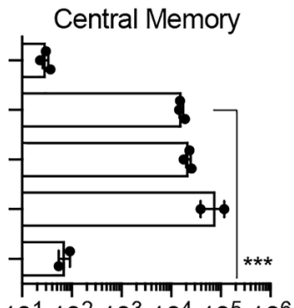

$10^{1} 10^{2} 10^{3} 10^{4} 10^{5} 10^{6}$

$\left[{ }^{3} \mathrm{H}\right]$ Thymidine Incorporation (Net CPM)

E

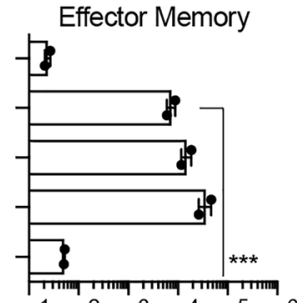

$10^{1} 10^{2} 10^{3} 10^{4} 10^{5} 10^{6}$

$\left[{ }^{3} \mathrm{H}\right]$ Thymidine Incorporation (Net CPM)

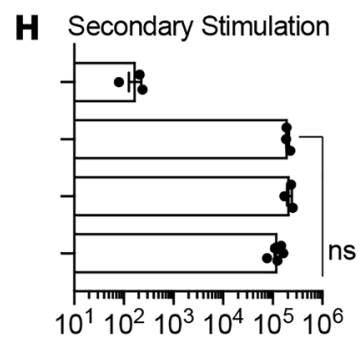

$\left[{ }^{3} \mathrm{H}\right]$ Thymidine Incorporation (Net CPM)

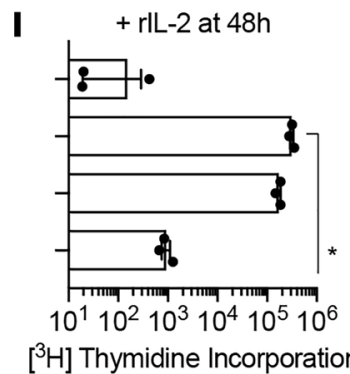

(Net CPM)

Figure 2. Suppressed T cells are viable and responsive to secondary stimulation. T cells (CD3+) and PMN were used in autologous coculture at 1:1. PMN and/or ascites supernatants (ASC; 50\% final well volume) were added to anti-CD3/CD28-stimulated T cells. After 72 hours of coculture, T cell proliferation was measured by $\left[{ }^{3} \mathrm{H}\right]$ thymidine incorporation (16-18 hours). (A) Results are consistent with soluble anti-CD3/CD28 Ab or anti-CD3/CD28 microbeads as T cell stimulus. (B) ASC $(n=31)$ were stratified into 3 categories based on the induction of a PMN suppressor phenotype, where $X$ equals a reduction in proliferation as compared with anti-CD3/CD28-stimulated T cells alone: suppressors (SUPP, line $3 ; X \geq 1 \log _{10}$ ), intermediate suppressors (INTERMED, line 2; 0.5 $\log _{10} \leq X<1 \log _{10}$ ), and nonsuppressors (NON-SUPP, line $1 ; X<0.5 \log _{10}$ ). SUPP-A and -B illustrate that a subset of ascites supernatants induced PMN suppressors $X \geq 2 \log _{10}$. Bars are representative of the stratification of suppressor status defined in Table 2. (C-E) PMN suppressor phenotype fully suppressed anti-CD3/CD28-stimulated naive (CD3+CD45RA+RO-CD62L') (C), central memory (CD3+CD45RA-RO+CD62L+) (D), and effector memory (CD3+CD45RA ${ }^{-} R 0^{+} \mathrm{C}^{+}$ D62 $\left.\mathrm{L}^{-}\right)(\mathbf{E})$ T cell populations $(n=2)$. (F) T cells were annexin-V-negative $(\geq 70 \%)$ after 72 -hour coculture with ASC $(n=3)$ and/or PMN. Fas ligand was added to stimulated T cells as a positive control for apoptosis. ( $\mathbf{G}$ and $\mathbf{H})$ Stimulated T cell proliferation was suppressed after 72 hours with ASC and PMN (G), but was restored after ASC removal and anti-CD3/CD28 restimulation $(n=5)(\mathbf{H})$. (I) Addition of rIL-2 $(100 \mathrm{IU})$ at 48 hours did not rescue T cell proliferation, as assessed at 72 hours. Symbols represent individual samples $(n)$, and bars represent \pm SEM. Statistical comparisons were by ANOVA with Tukey post hoc test $\left({ }^{*} P<0.05 ;{ }^{* *} P<0.001\right)$. Results were consistent between $\mathrm{CD}^{+}{ }^{+}$and $\mathrm{CD} 8^{+} \mathrm{T}$ cells.

To assess the effect of ER transport on the PMN suppressor phenotype, we pretreated PMN with brefeldin-A and an ER export inhibitor, Exo1. Both agents abrogated the PMN suppressor phenotype (Figure $4 \mathrm{G}$ ). We further evaluated the role of exocytosis using fusion proteins containing the TAT cell permeability sequence and either the soluble $\mathrm{N}$-ethylmaleimide-sensitive factor activating protein receptor 
A

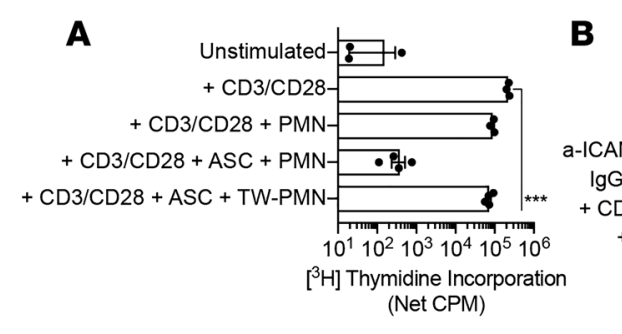

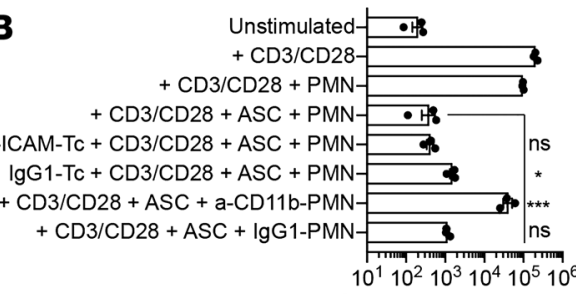

$\left[{ }^{3} \mathrm{H}\right]$ Thymidine Incorporation (Net CPM)

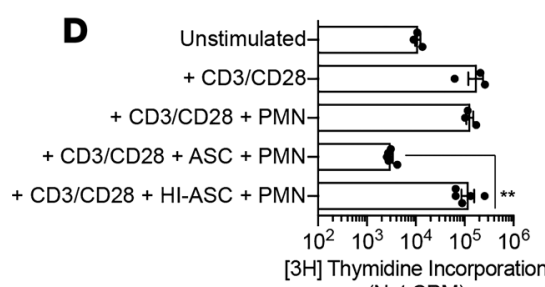

E
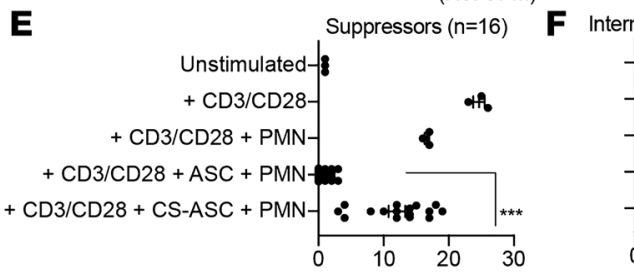

(Net CPM)

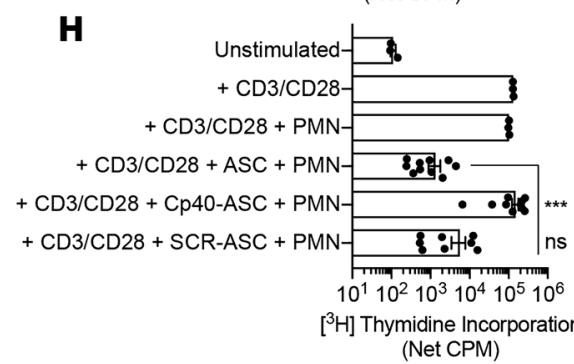

I

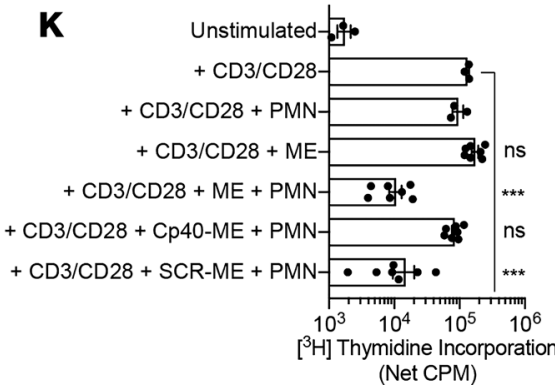

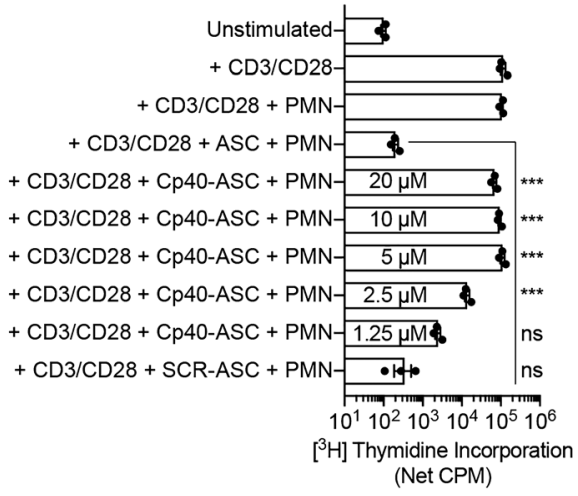

(Net CPM)
(n)

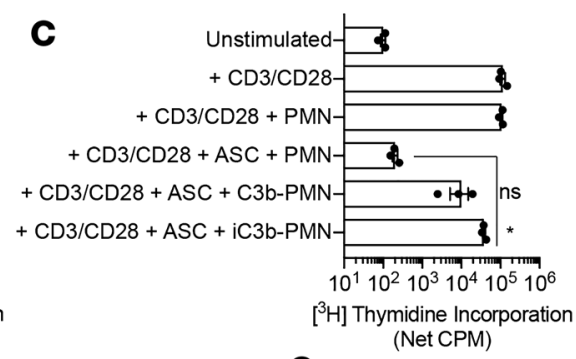

rmediate Suppressors $(n=8)$

G Non-Suppressors ( $(n=3)$
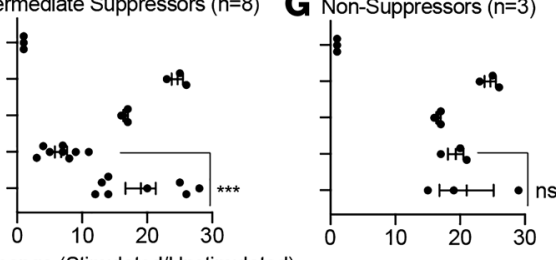

J

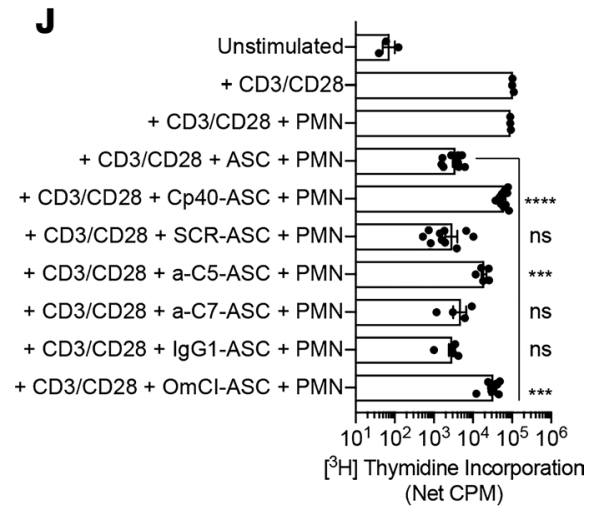

Figure 3. PMN suppressor phenotype requires contact between PMN and T cells, complement $\mathrm{C} 3$ activation, and complement receptor $3 . \mathrm{T}$ cells (CD3+) and PMN were used in autologous coculture at 1:1. PMN and/or ascites supernatants (ASC; 50\% final well volume) were added to anti-CD3/CD28-stimulated T cells. After 72 hours of coculture, T cell proliferation was measured by $\left.{ }^{3} \mathrm{H}\right]$ thymidine incorporation (16-18 hours). (A) T cells were stimulated with anti-CD3/CD28 in the bottom chamber. PMN and ASC added to the transwell insert did not suppress T cell proliferation $(n=4)$. (B) T cells treated with anti-ICAM-1 Ab (1-10 $\mu \mathrm{g})$ for 1 hour prior to coculture had no effect on proliferation. PMN treated with anti-CD11b Ab for 1 hour prior to coculture abrogated the suppressor phenotype. Treatment of T cells or PMN with IgG1 isotype $(1-10 \mu \mathrm{g})$ had no effect on proliferation $(n=5)$. (C) PMN pretreated with C3b or iC3b $(40-160 \mu \mathrm{g} / \mathrm{ml})$ prior to coculture were unable to induce the suppressor phenotype. (D) ASC were heat inactivated (HI-ASC; $\left.56^{\circ} \mathrm{C}, 1 \mathrm{hour}\right)$ prior to coculture and abrogated the PMN suppressor phenotype $(n=5)$. (E-H) Two formulations of compstatin, CS and Cp40, were used to inhibit C3 activation. Addition of CS $(250 \mu \mathrm{M})$ to ASC (CS-ASC) 2 hours prior to coculture with PMN and T cells abrogated the suppressor phenotype $(n=27)(E-C)$. Addition of Cp40 $(20 \mu \mathrm{M})$ to ASC (Cp40-ASC) 2 hours prior to coculture also abrogated the suppressor phenotype, while scramble peptide (SCR-ASC, $20 \mu \mathrm{M})$ had no effect $(n=10)(\mathbf{H})$. (I) A titration study showed that $5 \mu \mathrm{M}$ Cp40 was sufficient to fully abrogate the suppressor phenotype $(n=3)$. (J) ASC were pretreated with neutralizing $\mathrm{Ab}$ anti-C5 or $-\mathrm{C}$, or with $\mathrm{OmCl}$, a peptide inhibitor of $\mathrm{C5}$, prior to coculture. Anti- $\mathrm{C} 5$ and $\mathrm{OmCl}$ partially abrogated the suppressor phenotype, as compared with their respective controls, whereas anti-C7 did not affect the suppressor phenotype. (K) Malignant effusions (ME), including pleural fluid and ascites from patients with a number of metastatic cancers, induced the PMN suppressor phenotype, which was abrogated by Cp40 treatment in all of the tested samples ( $n=7$; Table 3 ). Symbols represent individual samples $(n)$, and bars represent \pm SEM. Statistical comparisons were by ANOVA with Tukey post hoc test or by Mann-Whitney $\left({ }^{*} P<0.05 ;{ }^{* *} P<0.01 ;{ }^{* *} P<0.001\right)$.

(SNARE) domain of syntaxin-4 or the N-terminal of soluble N-ethylmaleimide-sensitive factor activating protein 23 (SNAP23). SNARE decoys inhibit stimulated exocytosis of secretory vesicles and secondary and tertiary granules, but not primary granules, in $\operatorname{PMN}(36,37)$. PMN pretreated with the SNARE decoys for SNAP23 and syntaxin- 4 abrogated the PMN suppressor phenotype, while TAT fusion proteins with GST, a specificity control, had no effect (Figure 4H). These results show that SNARE-dependent exocytosis is required for the PMN suppressor phenotype. 
Table 3. Clinical characteristics of patients with malignant effusions that induced the PMN suppressor phenotype in a complement C3-dependent mechanism

\begin{tabular}{lc}
\hline Demographics of patients with malignant effusions other than EOC ascites & 7 \\
$n$ & 68.4 \\
Age, mean (year) & $5: 2$ \\
Sex (Female/Male) & 1 \\
Type of malignant effusions & 6 \\
$\quad$ Peritoneal sscites & \\
$\quad$ Pleural effusion & 2 \\
Metastatic cancer & 2 \\
$\quad$ Ovarian & 1 \\
$\quad$ Lung & 1 \\
$\quad$ Pancreatic ${ }^{A}$ & 1 \\
$\quad$ Gl & 3 \\
$\quad$ Lymphoma & 4 \\
Newly diagnosed & \\
Received prior treatment (e.g., surgery, chemotherapy, & \\
immunotherapy) & \\
${ }^{A}$ Ascites. Gl, gastrointestinal. & \\
\end{tabular}

Activation with $\mathrm{fMLF}$ induced PMN to inhibit $\mathrm{T}$ cell responses through a mechanism requiring hydrogen peroxide generation (27). Therefore, we asked whether pretreatment of PMN prior to ascites exposure would desensitize PMN. We observed that activation with fMLF prevented induction of the PMN suppressor phenotype, indicative of heterologous desensitization (Figure 4I). In addition, PMN pretreated with thapsigargin (THG; inhibitor of $\mathrm{Ca}^{2+}$ mobilization) abrogated the PMN suppressor phenotype. To determine the role of PMN ROS in suppressing T cell proliferation, we evaluated the effect of the ROS scavenger, $\mathrm{N}$-acetyl cysteine (NAC), and diphenyleneiodonium (DPI), which is a small molecule flavocytochrome inhibitor of NADPH oxidase, the major source of PMN ROS generation. Addition of NAC to cocultures did not reverse suppression (Supplemental Figure 5A), while pretreating PMN with DPI did abrogate the PMN suppressor phenotype (Figure 4I). These results are conflicting regarding the role of ROS generation in the PMN suppressor phenotype; further studies using PMN from patients with chronic granulomatous disease (CGD), an inherited disorder of the phagocyte NADPH oxidase, will delineate the role of NADPH oxidase in the PMN suppressor function.

Release of arginase-1 from tertiary granules can also suppress T cells (28). Addition of L-arginine to the cocultures had no effect on $\mathrm{T}$ cell suppression (Supplemental Figure 5A), arguing against arginase-1 mediating the PMN suppressor phenotype. We previously observed that ascites stimulated NET generation (30), and Lee et al. (38) recently demonstrated that NETs facilitated premetastatic niche formation in murine EOC. However, pretreatment of PMN with CI-amidine, an inhibitor of protein arginine deiminase 4 required for NET generation, or addition of DNase I to the cocultures to degrade NETs had no effect on the PMN suppressor phenotype (Supplemental Figure 5A), suggesting that the mechanism is independent of NETs. Wong et al. (39) recently showed that IFN- $\gamma$ and TNF- $\alpha$ synergize to induce cyclooxygenase-2 (COX2) in the EOC TME, which in turn hyperactivates MDSC and leads to overexpression of the immunosuppressive enzyme, indoleamine-2,3-dioxygenase (IDO). We observed that addition of indomethacin, a nonselective COX inhibitor (Supplemental Figure 5B); zileuton, an inhibitor of 5-lipoxygenase (Supplemental Figure 5C); and 1-methyl-DL-tryptophan, an inhibitor of IDO1/2 (Supplemental Figure 5D) to the cocultures had no effect on the PMN suppressor phenotype, indicating that the arachidonic acid pathway and IDO are likely not playing a role. Finally, high levels of TGF- $\beta$ are present in EOC ascites (40), and TGF- $\beta$ signaling can skew TAN to a suppressive N2 phenotype (24). We observed that anti-TGF- $\beta$ receptor $1 \mathrm{Ab}$ did not abrogate the PMN suppressor phenotype (Supplemental Figure $5 \mathrm{E})$. Together, these results show that multiple PMN effector pathways are required for mediation of the suppressor phenotype in mature PMN that are distinct from those associated with MDSC or N2 TAN.

Ascites induces robust protein synthesis in PMN that is required for the suppressor phenotype. We asked whether protein synthesis in PMN was required for the suppressor phenotype. Pretreating PMN with the protein 
A Time (min) 0

CD63 ( $1^{\circ}$ granules)

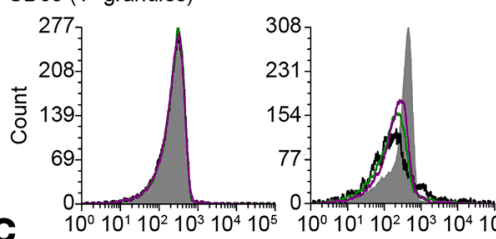

30

60

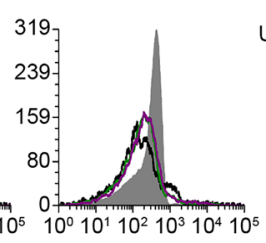

CD66b $\left(2^{\circ}, 3^{\circ}\right.$ granules $)$

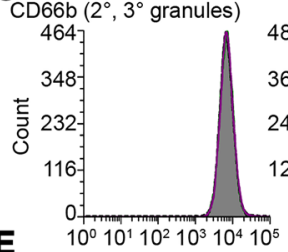

ED35 (secretory vesicles)
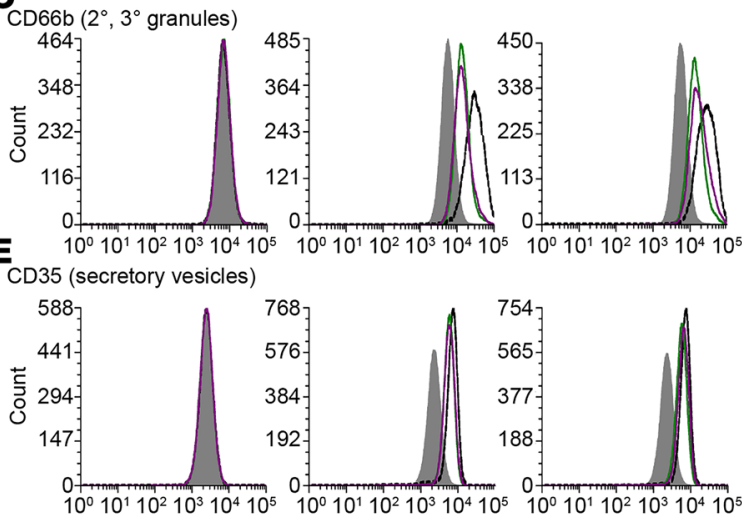

$\mathrm{MFI}$ of $\mathrm{CD} 45^{+} \mathrm{CD} 15^{+}$cells

G

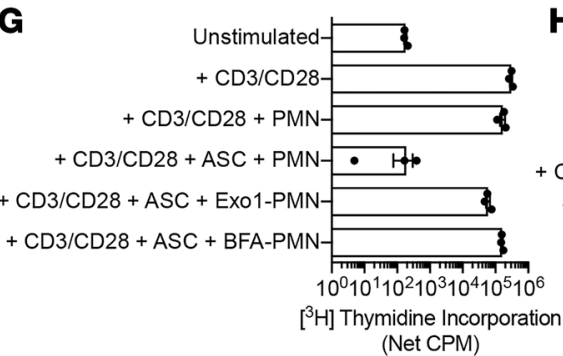

H
B $\begin{gathered}\text { Time }(\min ) \quad 0 \\ \operatorname{CD} 63\left(1^{\circ} \text { granules }\right)\end{gathered}$

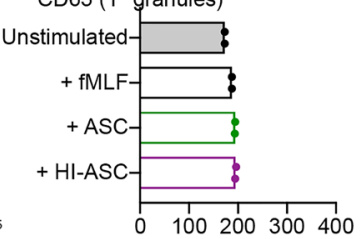

D CD66b $\left(2^{\circ}, 3^{\circ}\right.$ granules $)$

Unstimulated-?

$+\mathrm{fMLF}=?$

$+\mathrm{ASC}-?$

$+\mathrm{HI}-\mathrm{ASC}-\mathrm{B}$

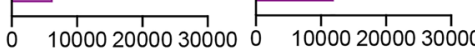

F CD35 (secretory vesicles)

Unstimulated-?

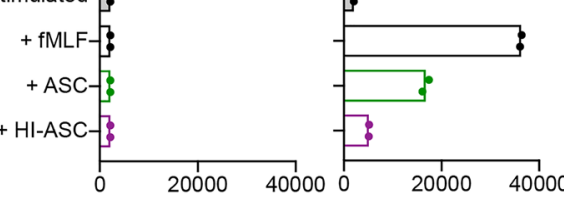

$\mathrm{MFI}$ of $\mathrm{CD} 45^{+} \mathrm{CD} 15^{+}$cells
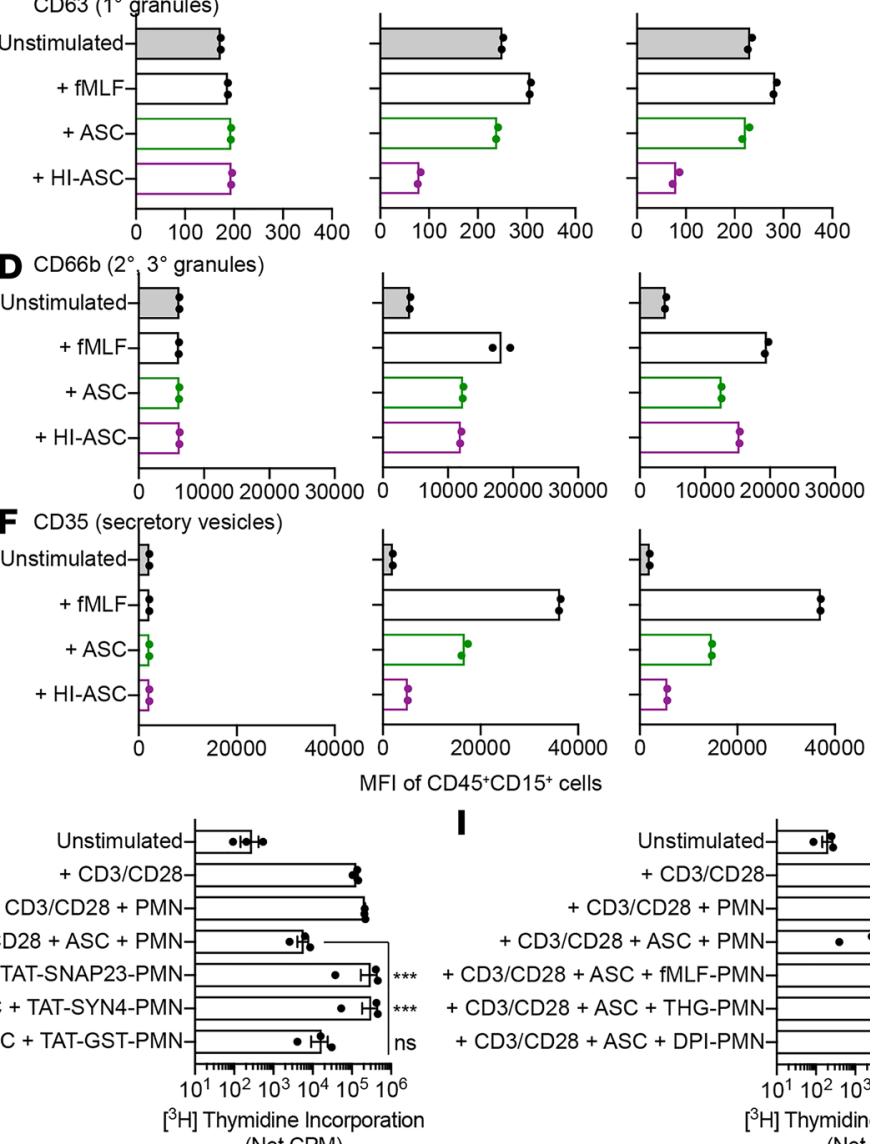
(Net CPM)

I

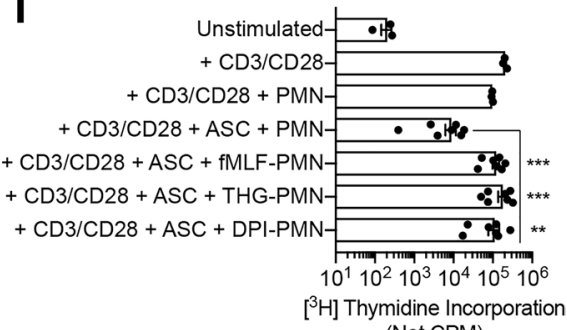
(Net CPM)

Figure 4. PMN suppressor phenotype requires SNARE transport and Ca ${ }^{2+}$ mobilization, and it is abrogated by desensitization with fMLF. (A-F) PMN were treated with media, fMLF $(100 \mathrm{nM})$, ASC $(n=3)$, or heat-inactivated ASC (HI-ASC; $n=3$ ) and assessed for markers of membrane fusion with primary (CD63), secondary and tertiary (CD66b) granules, and secretory vesicles (CD35) at 0, 30, and 60 minutes. PMN were gated on CD45+CD15+. (A, C, and E) The MFI overlays are representative; unstimulated PMN in media, gray solid; fMLF, black dashed line; ASC, green line; HI-ASC, purple line. (B, D, and F) MFI quantification. (G-I) T cells (CD3+) and PMN were used in autologous coculture at 1:1. PMN and/or ascites supernatants (ASC; $50 \%$ final well volume) were added to anti-CD3/CD28-stimulated T cells. After 72 hours of coculture, T cell proliferation was measured by [ $\left.{ }^{3} \mathrm{H}\right]$ thymidine incorporation (16-18 hours). (G) Pretreatment with brefeldin-A (BFA; $1-10 \mu \mathrm{g} / \mathrm{ml}$ ) or ER export inhibitor 1 (Exo1; $20-75 \mu \mathrm{M}$ ) abrogated the suppressor phenotype, indicating a requirement for exocytosis $(n=3)$. (H) PMN pretreated with TAT-SNAP23 (0.6 $\mu$ g) or TAT-SYN4 (0.6 $\mu$ g) abrogated the PMN suppressor phenotype. TAT-GST (0.6 $\mu \mathrm{g})$ used as a specificity control had no effect $(n=3)$. (I) PMN pretreated with fMLF $(100 \mathrm{nM})$, thapsigargin (THG, $1 \mu \mathrm{M})$, or diphenyleneiodonium $(\mathrm{DPI}, 1 \mu \mathrm{M})$ abrogated the PMN suppressor phenotype $(n=7)$. Symbols represent individual samples $(n)$, and bars represent \pm SEM. Statistical comparisons were by ANOVA with Tukey post hoc test $\left({ }^{* *} P<0.01 ;{ }^{* *} P<0.001\right)$.

synthesis inhibitors, puromycin and actinomycin $\mathrm{D}$, resulted in variable abrogation of the suppressor phenotype (Figure 5A). Based on these results, we explored the effect of ascites on protein production in PMN. We exposed PMN to media, ASC, or PK-ASC for 30 and 60 minutes and PMN subsequently underwent proteomics analysis. Unique protein groups (1,935 groups) were quantified with $\geq 2$ peptides per protein and a $<2 \%$ missing data rate on the protein level. Proteome patterns were similar at 30- and 60 -minute time points and showed prominent discrepancies between PMN exposed to ascites versus PK-ASC (Figure 5B). The PK-ASC-exposed PMN displayed a proteome pattern more similar to PMN exposed to media. Under the selected cutoff thresholds $(>1.5$-fold protein change, $P<0.05)$ at 30 and 60 minutes, 630 and 638 proteins exhibited significant changes in the ascites groups, while only 160 and 195 proteins were significantly changed in the PK-ASC groups, respectively (Figure 5C). Notably, 175 and 173 proteins were exclusively changed with 30- and 60-minute ascites exposure, respectively. Gene ontology analysis of significant proteins showed enrichment of multiple classes of proteins with diverse biological functions in the ascites-exposed PMN (Figure 5D). Kyoto Encyclopedia of Genes and Genomes (KEGG) pathway (https://www.genome.jp/kegg/pathway.html) analysis showed that the transcription factors, STAT3 and its target PU.1, were highly upregulated in PMN exposed to ascites, as compared with PMN exposed to 
media $(P=0.01$ and $P=0.002$, respectively). Ascites led to increased levels of several granular constituents, including myeloperoxidase $(P=0.005)$, neutrophil elastase $(P=0.0001)$, cathepsin $\mathrm{G}(P=0.001)$, defensin $1(P=0.02)$, defensin $3(P=0.001)$, lysozyme C $(P=0.03)$, and MMP9 $(P=0.04)$. In addition, ascites exposure led to increased levels of multiple complement pathway and signaling components, including C1r $(P=0.003)$, C1q receptor $(P=0.01)$, C3 $(P<0.0001)$, C5 $(P<0.0001)$, C9 $(P<0.0001)$, properdin $(P<0.001)$, complement factor B $(P<0.0001)$, and complement factor D $(P<0.0001)$, as well as CR1 (CD35, $P<0.0001)$ and CR3 (CD18, $P=0.005$; CD11b, $P<0.0001)$. Ascites also led to decreased levels of a smaller subset of proteins involved in protein folding, microtubule-based processes, and response to ROS, including gp91 ${ }^{\text {phox }}(P=0.01)$, SOD $(P<0.0001)$, COX2 $(P<0.0001)$, NADPH-dependent carbonyl reductase $(P<0.0001)$, and promyelocytic leukemia protein (PML, $P=0.03)$. These results suggest that ascites induces synthesis of multiple classes of proteins in PMN and that protein synthesis is required for the PMN suppressor phenotype.

PMN suppressor phenotype inhibits stimulated naive T cell activation without inducing exhaustion marker upregulation and without affecting antigen-specific CTL killing. To further delineate the effects of the PMN suppressor phenotype on $\mathrm{T}$ cell immunity, we evaluated markers for $\mathrm{T}$ cell activation and exhaustion in cocultures. The proportion of CD62L-expressing T cells decreased after 24 hours of anti-CD3/CD28 stimulation as compared with baseline, a characteristic of newly activated $\mathrm{T}$ cells; this early reduction in CD62L expression was not observed with ascites and/or PMN (Figure 6A). The proportion of T cells expressing CD69 (Figure 6B), CD40L (Figure 6C), and CD107a (Figure 6D) increased with stimulation as compared with baseline (characteristic of newly activated $\mathrm{T}$ cells); in each case, upregulation was inhibited in cocultures with ascites and PMN. In addition, anti-CD3/CD28 stimulation upregulated the expression of exhaustion markers PD-1, LAG-3, and CTLA-4 on T cells (Figure 6, E-J). Coculture with ascites or PMN alone had more variable effects, while the combination of ascites and $\mathrm{PMN}$ prevented anti-CD3/CD28-stimulated upregulation of PD-1, LAG-3, and CTLA-4. Finally, while Cp40-ASC abrogated the PMN suppressor phenotype and enabled robust anti-CD3/CD28-stimulated T cell proliferation, Cp40-ASC did not result in upregulation of PD-1 or LAG-3 on T cells (Figure 6, K and L), while CTLA-4 expression was upregulated relative to unstimulated $\mathrm{T}$ cells (Figure $6 \mathrm{M}$ ).

Next, we evaluated the transcriptional control of $\mathrm{CD}^{+}$effector differentiation, as measured by the upregulation of T-bet and eomesodermin (Eomes) (41). T-bet expression is associated with CTL differentiation, while Eomes expression is associated with memory $\mathrm{T}$ cell differentiation. By gating on $\mathrm{CD}^{+} \mathrm{C}-$ $\mathrm{D}^{+}{ }^{+} \mathrm{CCR} 7^{-} \mathrm{T}$ cells (Supplemental Figure $6 \mathrm{~A}$ ), anti-CD3/CD28 stimulation increased the proportion of Eomes $^{+} \mathrm{T}-$ bet $^{\mathrm{hi}} \mathrm{T}$ cells by 24 hours, as compared with unstimulated cells, and this increase contracted by 96 hours (Supplemental Figure 6, B-E). T cells cocultured with ascites, and PMN phenocopied the Eomes $^{+}$T-bet ${ }^{\text {lo }}$ signature of unstimulated cells (Supplemental Figure 6, F and G), indicating that the PMN suppressor phenotype inhibits differentiation into $\mathrm{CD} 8^{+}$effector T cells. Ascites and/or PMN reduced the proportion of anti-CD3/CD28-stimulated CD8 ${ }^{+} \mathrm{T}$ cells expressing IFN- $\gamma$ (Figure $6 \mathrm{~N}$ ). In addition, ascites or PMN alone both reduced anti-CD3/CD28-stimulated production of IL-2 by T cells at 24 and 72 hours, while cocultures with ascites and PMN completely abrogated T cell IL-2 production (Figure 6, O and P). Together, these results show that the PMN suppressor phenotype inhibits T cell activation independently of upregulation of exhaustion marker expression and has broad inhibitory effects on $\mathrm{T}$ cell activation, including the suppression of effector differentiation and cytokine responses.

Finally, we evaluated whether the PMN suppressor phenotype affected tumor cell lysis. NY-ESO-1specific $\mathrm{CD}^{+} \mathrm{T}$ cells from patients who received NY-ESO-1 vaccination were amplified in vitro and NY-ESO- $1_{157-165}$-specific CD8 ${ }^{+}$T cells were isolated as described (42). Using NY-ESO- $1_{157-165}$-specific CD8 ${ }^{+}$ CTL and tumor cell (SK29) targets preloaded with NY-ESO-1 ${ }_{157-165}$ peptide, we observed that PMN and/or ascites had no effect on antigen-specific cytotoxicity (Figure 6Q). Together, these results show that the PMN suppressor phenotype suppressed the expansion and activation of T cells without affecting CTL activity.

PMN suppressor phenotype inhibits the expansion of TCR-engineered CTL. To further understand how the PMN suppressor phenotype may be a barrier to immunotherapy, we evaluated the effect of cocultures with ascites and PMN on CTL with engineered TCR that recognize the tumor antigen, NY-ESO-1, and are in development for adoptive cellular therapy. Engineered CTL are activated during the expansion process prior to use in cocultures, accounting for the higher baseline proliferation observed in unstimulated cells and the modest increase in proliferation observed in anti-CD3/CD28-stimulated cells (Figure 7A). The PMN suppressor phenotype inhibited stimulated proliferation of CTL below unstimulated levels, while 
A

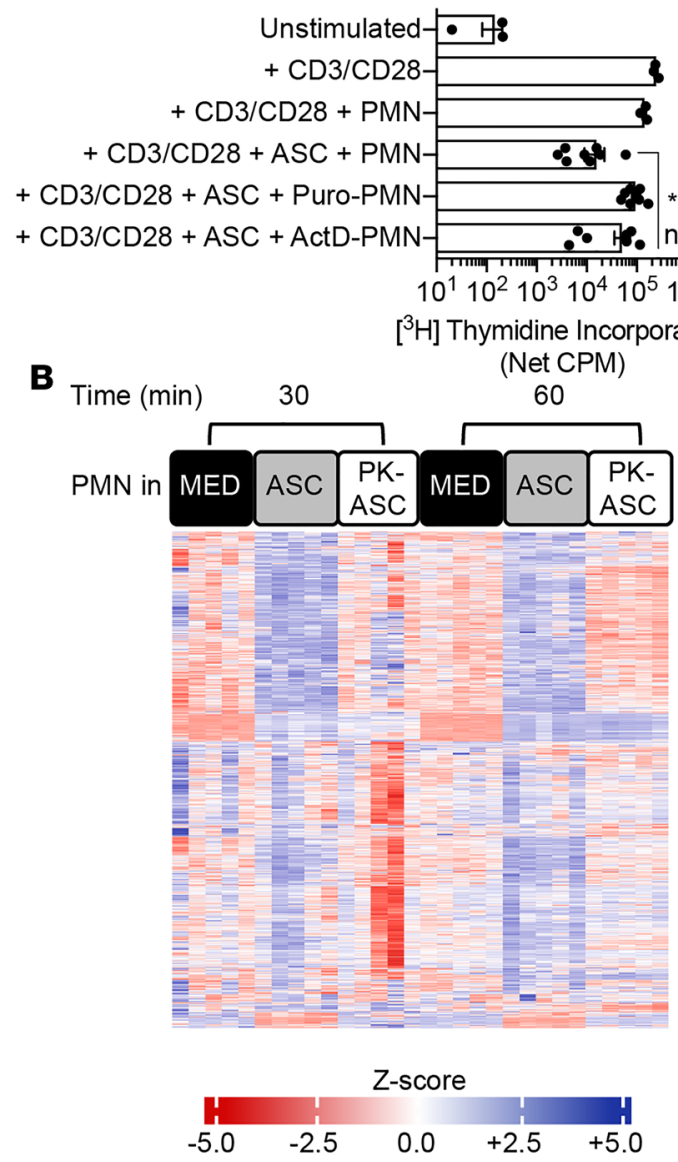

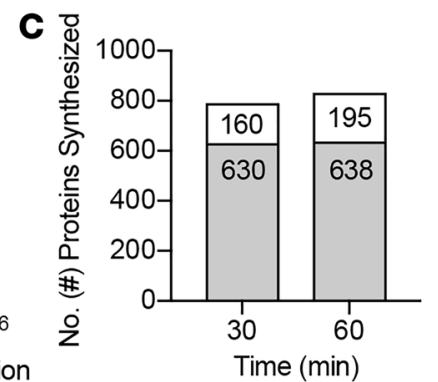
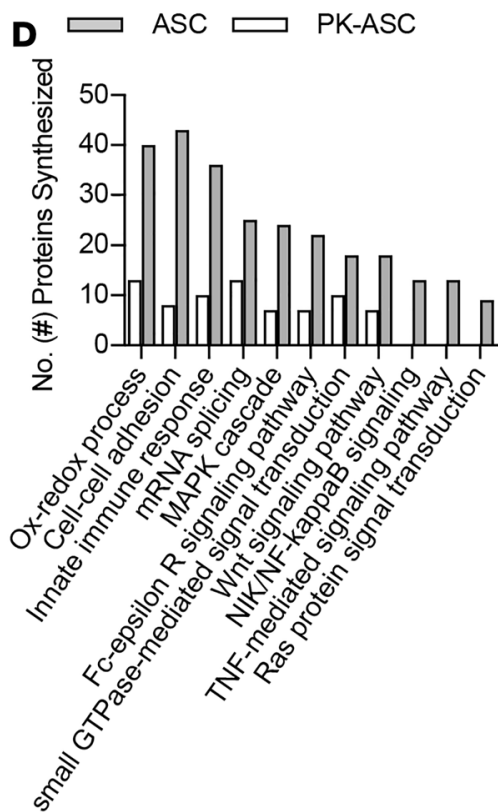

Figure 5. Ascites induces robust de novo protein synthesis in PMN, which is required for the suppressor phenotype. (A) PMN pretreated with puromycin $(1 \mu \mathrm{g})$ for 1 hour prior to coculture abrogated the PMN suppressor phenotype, while D-actinomycin $(1 \mu \mathrm{g})$ pretreatment had more variable effects $(n=8)$. (B-D) PMN were exposed to media, ASC, or proteinase K-digested ASC (PK-ASC) for 30 or 60 minutes in 5 replicates per condition per time point. PMN were washed and frozen as dry pellets for proteomics analysis. (B) Heatmap showing that the protein profiles of PMN exposed to ASC have higher Z-scores than either PMN exposed to media or PK-ASC at 30 and 60 minutes. (C) The number of changed proteins ( $y$ axis) in PMN exposed to ASC is significantly greater than in PMN exposed to PK-ASC $(P=0.02)$; there was no significant difference between 30 or 60 minutes. (D) Gene ontology analysis shows that ASC induced new synthesis of multiple classes of proteins in PMN. Symbols represent individual samples $(n)$, and bars represent \pm SEM. Statistical comparisons were by ANOVA with Tukey post hoc test $\left.{ }^{* *} P<0.01\right)$.

neither ascites nor PMN alone had an effect on proliferation. PK-ASC abrogated the PMN suppressor phenotype, while MD-ASC had no effect, consistent with data in primary T cells. In contrast to cocultures with primary $\mathrm{T}$ cells where rIL-2 did not reverse $\mathrm{T}$ cell suppression, addition of rIL-2 to cocultures with engineered CTL at 48 hours completely restored proliferation, suggesting that mechanisms for reversal of the PMN suppressor phenotype depends on activation status of the T cells (Figure 7B). IFN- $\gamma$ expression was reduced to a similar level after cocultures with PMN and/or ascites (Figure 7C). These results point to the PMN suppressor phenotype within the TME as a potential barrier to adoptive cellular therapy.

Postoperative drainage fluid induces the PMN suppressor phenotype. Finally, we questioned whether the PMN suppressor phenotype was specific to the TME or instead a more general response to injury. We evaluated whether postoperative peritoneal fluid collected from a surgical drain 1 day after primary surgery for EOC would induce the PMN suppressor phenotype. In contrast to ascites collected prior to surgery, which contained a mixed WBC population, postoperative drainage fluid indicated a neutrophilic peritonitis (Supplemental Figure 7, A-E). The numbers of cells in the postoperative drainage fluid were insufficient for suppression studies. Therefore, we compared the capacity of paired ASC and postoperative drainage supernatants to induce the PMN suppressor phenotype. The debulking statuses of these patients were R0 ( 3 of 7), defined as no macroscopic residual tumor, or optimal (4 of 7), 

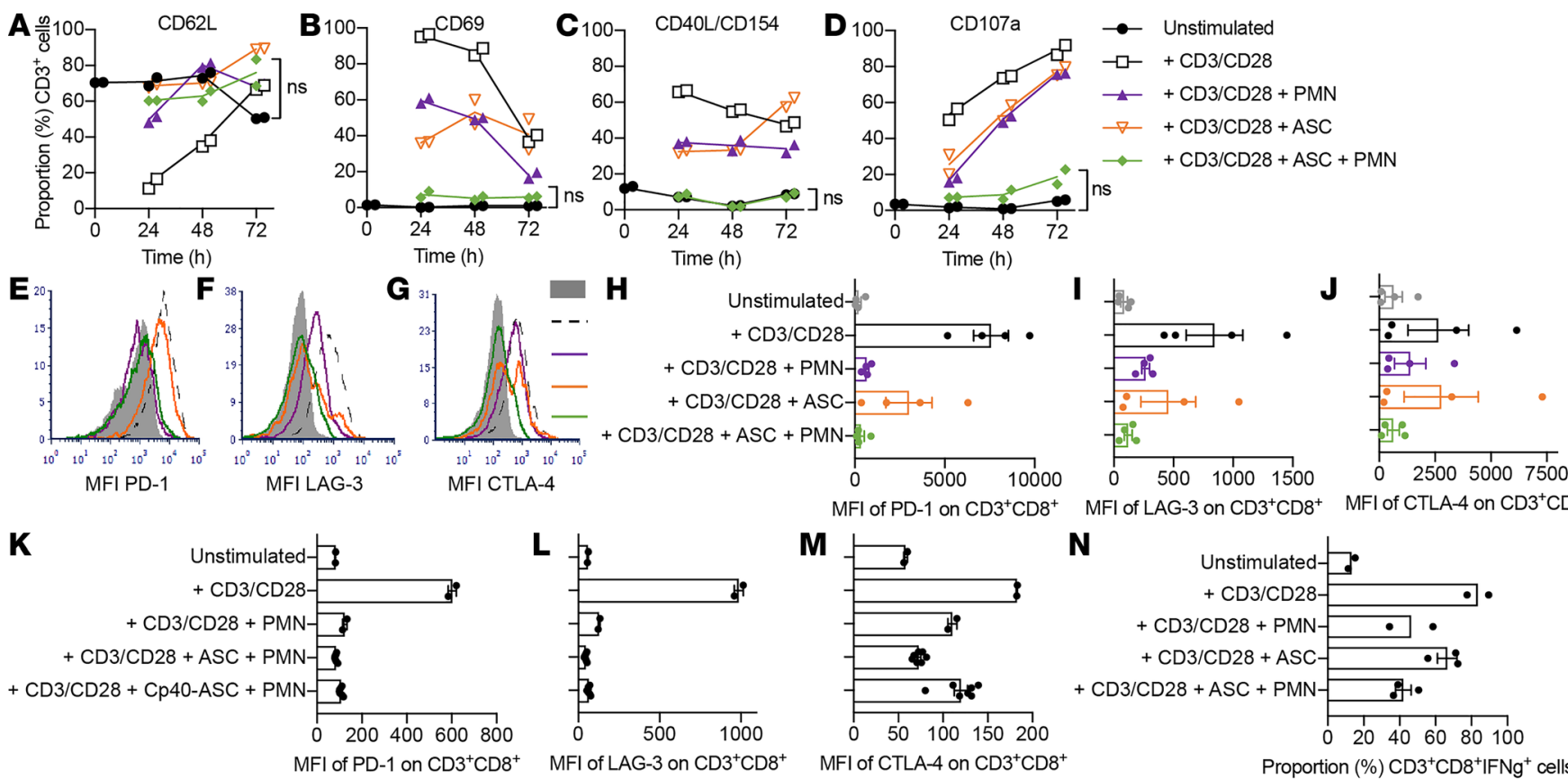

$\mathbf{L}$

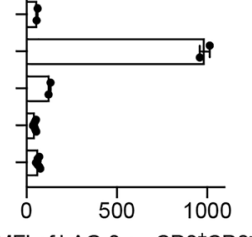

MFI of LAG- 3 on $\mathrm{CD}^{+}{ }^{+} \mathrm{CD} 8^{+}$
M

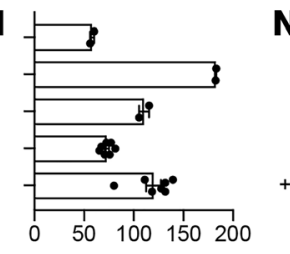

MFI of CTLA- 4 on $\mathrm{CD}^{+} \mathrm{CD}^{+}$
N

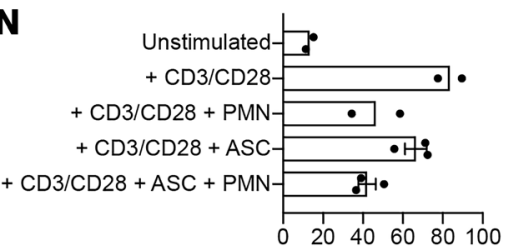

Proportion (\%) $\mathrm{CD}^{+} \mathrm{CD}^{+} \mathrm{IFNg}^{+}$cells
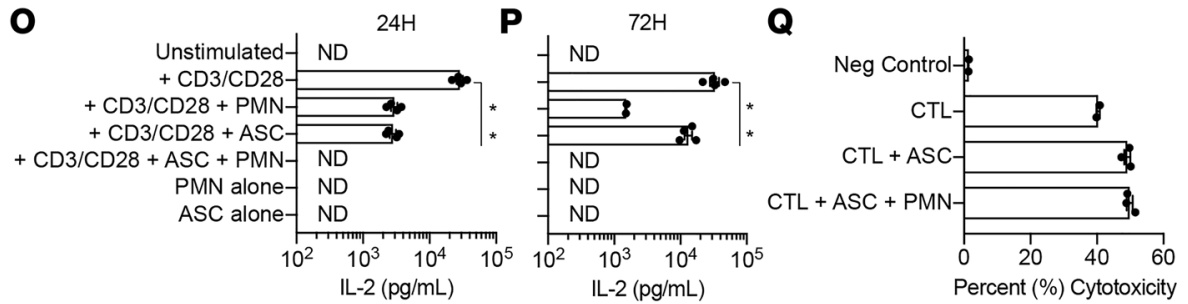

Figure 6. Combination of ascites and PMN prevents $\mathrm{T}$ cell activation and is independent of exhaustion. T cells (CD3+) and PMN were used in autologous coculture at 1:1. PMN and/or ascites supernatants (ASC; 50\% final well volume) were added to anti-CD3/CD28-stimulated T cells. At 24, 48, and 72 hours, T cells were analyzed for surface and intracellular expression of markers for activation, costimulation, and function. Surface expression of (A) CD62L, (B) CD69, (C) CD40L, and (D) CD107a were evaluated at baseline (on $y$ axis) and at 24, 48, and 72 hours ( $n=2$ ). (E-M) PD-1, LAG-3, and CTLA-4 expression was evaluated $(n=4)$. CD8 ${ }^{+}$T cells at 72 hours are represented here in MFI overlays $(\mathbf{E}-\mathbf{C})$ and quantification (H-J); unstimulated T cells in media, gray solid; anti-CD3/CD28-stimulated, black dashed line; anti-CD3/CD28-stimulated + PMN, purple solid line; anti-CD3/CD28-stimulated + ASC, orange solid line; anti-CD3/CD28-stimulated + ASC + PMN, green solid line. (K-M) Stimulated T cell expression of PD-1 and LAG-3 after coculture with Cp40-ASC and PMN was unaffected as compared with unstimulated, but CTLA-4 showed an upwards trend $(n=6)$. (N) At 72 hours, intracellular expression of IFN- $\gamma$ was reduced as compared with stimulated alone $(n=3)$. ( $\mathbf{0}$ and $\mathbf{P})$ Combination of ASC and PMN reduced anti-CD3/CD28-stimulated T cell IL-2 levels $(\mathrm{pg} / \mathrm{ml})$ in supernatants to ND after $24(\mathbf{O})$ and 72 hours $(\mathbf{P})$ of coculture $(n=4)$. Background levels of PMN or ASC were nondetectable (ND). (Q) CTL activity of NY-ESO-1 $1_{157-165}$-specific CD8 ${ }^{+}$T cells directed at SK29 target cells pulsed with the NY-ESO-1 peptide was unaffected by coculture with ASC and/or PMN $(n=3)$. Symbols represent individual samples $(n)$, and bars represent \pm SEM. Statistical comparisons were by Mann-Whitney ( $\left.{ }^{*} P<0.05\right)$. Results were consistent between $\mathrm{CD}^{+}$and $\mathrm{CD} 8^{+} \mathrm{T}$ cells.

defined by remaining disease $0.1-1 \mathrm{~cm}$. Similar to ascites, postoperative drainage supernatants were not suppressive alone but induced PMN to suppress anti-CD3/CD28-stimulated $\mathrm{T}$ cell proliferation (Supplemental Figure 7F).

To further probe whether the PMN suppressor phenotype was specific to the TME, we tested whether ASC from patients with cirrhosis and without cancer had the ability to induce the PMN suppressor phenotype. We observed T cell suppression in 1 of 3 samples tested (Supplemental Figure 7G). These findings support the notion that inflammation and injury, whether resulting from the TME or other pathologic conditions, can induce the PMN suppressor phenotype.

\section{Discussion}

Our results show that, in patients with newly diagnosed advanced EOC, circulating PMN are not intrinsically suppressive but acquire a suppressor phenotype once recruited to the TME. ASC induced PMN to suppress stimulated $\mathrm{T}$ cell proliferation, activation, and cytokine responses but did not affect CTL activity. These findings suggest that, while the PMN suppressor phenotype will not affect the CTL activity of effector 
A

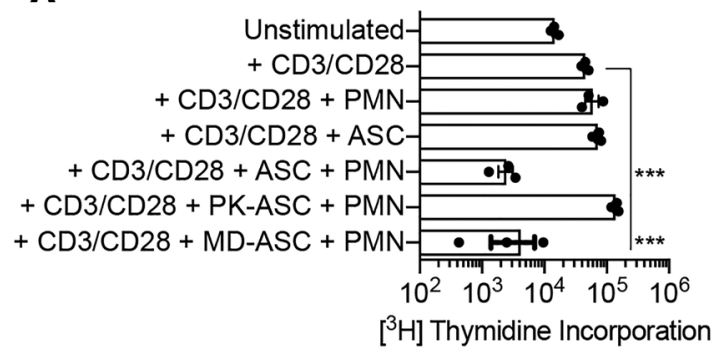

(Net CPM)

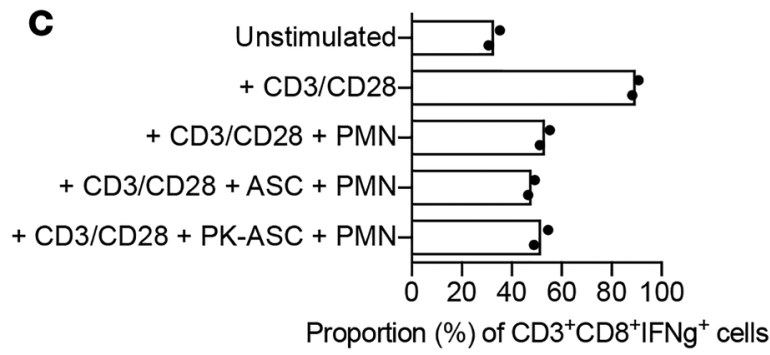

B

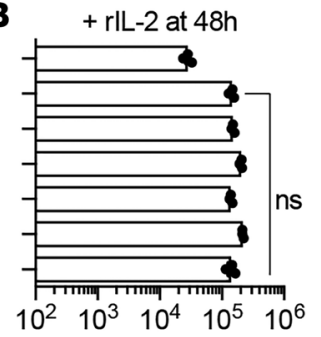

$\left.{ }^{3} \mathrm{H}\right]$ Thymidine Incorporation

(Net CPM)

Figure 7. PMN suppressor phenotype inhibits engineered effector $\mathrm{T}$ cell activation but not antigen-specific cytotoxicity. Engineered CTL expressing NY-ESO-1-specific TCR (TCR-CTL) are candidates for adoptive cell therapy in EOC. TCR-CTL and PMN were used in coculture at 1:1. PMN and/or ascites supernatants (ASC; $50 \%$ final well volume) were added to anti-CD3/CD28-stimulated TCR-CTL. After 72 hours of coculture (A) or after rIL-2 (100 IU) addition at 48 hours (B), CTL proliferation was measured by $\left[{ }^{3} \mathrm{H}\right]$ thymidine incorporation (16-18 hours) $(n=3)$. (A) ASC and MD-ASC rendered PMN suppressive to TCR-CTL, while PK-ASC had no effect. (B) Addition of rIL-2 reversed suppression. (C) IFN- $\gamma$ expression was reduced with PMN and/or ASC or PK-ASC, as measured by flow cytometry after 72 hours of coculture $(n=2)$. Symbols represent individual samples $(n)$, and bars represent \pm SEM. Statistical comparisons were by ANOVA with Tukey post hoc test $\left({ }^{* *} P<0.001\right)$.

T cells, the phenotype will prevent the expansion of these CTL in the TME. The PMN suppressor phenotype inhibited $\mathrm{T}$ cell proliferation in stimulated naive, central memory, and effector memory $\mathrm{T}$ cells, as well as in CTL with engineered TCR. Mature PMN fully recapitulated the suppressor phenotype attributed to granulocytic MDSC and N2 TAN. Although the distinction between granulocytic MDSC and N2 TAN is debated (19), the common feature is a circulating population of suppressor granulocytes $(20,43)$, while the PMN suppressor phenotype that we identified is acquired in the TME and dependent on several PMN effector functions. In addition, targeting TGF- $\beta$ signaling did not abrogate the phenotype, suggesting that this newly identified PMN suppressor phenotype is distinct from TGF- $\beta$-driven N2 polarization. Moreover, malignant effusions from patients with various metastatic cancers also induced the C3-dependent PMN suppressor phenotype, supporting the generalizability of these findings. Together, these results point to mature PMN impairing T cell expansion and activation in the TME and identify a number of therapeutic targets to abrogate this barrier to antitumor immunity. Of the pathways driving the PMN suppressor phenotype, complement activation and signaling are likely to be the most promising therapeutic targets, given the availability of approved and investigational inhibitors of complement components.

The role of complement activation in cancer is complex (44) and includes both pathways that can limit or worsen tumor growth. Complement can kill tumor cells through complement-dependent cytotoxicity, which is an important mechanism for tumor killing by monoclonal Ab. C3d enhanced antitumor immunity by increasing tumor-infiltrating $\mathrm{CD} 8^{+} \mathrm{T}$ cells, depleting Tregs, and suppressing PD-1 expression on $\mathrm{T}$ cells (45). In addition, complement activation in tumor vasculature facilitated $\mathrm{T}$ cell homing and control of tumor in adoptive cellular therapy (46). However, tumor cells can secrete complement proteins that stimulate tumor growth and epithelial mesenchymal transition $(47,48)$. Complement activation can also promote platelet-granulocyte aggregation, thrombosis, and NETosis $(49,50)$. CR3 signaling was associated with suppression of NK cell function and increased growth of syngeneic melanoma (51). The C5aR1 peptide antagonist, PMX-53, improved the efficacy of Paclitaxel chemotherapy and was associated with the increased accumulation and cytotoxic function of $\mathrm{CD} 8^{+}$effector memory $\mathrm{T}$ cells (52). In additional studies, the combination of C5a and anti-PD-1 blockade reduced tumor growth and metastasis (53). Most relevant to EOC, genetic and pharmacologic inhibition of complement blocked tumor growth through pathways dependent on VEGF 
and neovascularization in transgenic mice that develop EOC (54). Our results using human samples add to this body of literature by demonstrating a C3-dependent PMN suppressor phenotype induced by malignant effusions, and they further support the concept of targeting complement to enhance immunotherapy.

A strength of our study was the use of ascites from patients, rather than tumor-conditioned media or tumor-bearing mice. Though the majority of ascites induced the PMN suppressor phenotype $\left(\geq 1 \log _{10}\right.$ reduction of anti-CD3/CD28-stimulated $\mathrm{T}$ cell proliferation), this was not a universal finding. Our results point to soluble, heat-labile proteins, specifically complement, in ascites inducing the PMN suppressor phenotype. Future studies involving fractionation and proteomics analysis of suppressor and nonsuppressor ascites may delineate multiple proteins required for rendering PMN suppressive. A limitation of our study is that the small number of patients precluded an analysis of whether PMN accumulation in ascites or the capacity of ascites to induce the PMN suppressor phenotype correlated with outcomes.

PMN are easily activated when separated from whole blood. Erythrocyte sialoglycoproteins suppress PMN activation, and erythrocyte removal during purification can release this inhibitory function (55). Negorev et al. (56) reported that PMN contaminated peripheral blood mononuclear cell (PBMC) fractions after density gradient centrifugation methods were used to isolate granulocytic MDSC and may artefactually suppress $\mathrm{T}$ cell proliferation in certain cellular assays, including those that use microbeads for stimulation. We observed negligible to no effect of PMN alone in T cell suppression studies, while PMN combined with ASC resulted in dramatic suppression of $\mathrm{T}$ cell proliferation, frequently to unstimulated levels. The effect was observed with both anti-CD3/CD28 microbeads and soluble anti-CD3/CD28 Ab. Finally, we used a high standard for defining suppression, defining it as $\geq 1 \log _{10}$ reduction in anti-CD3/CD28-stimulated $\mathrm{T}$ cell proliferation, which was well above any background effects observed with PMN alone.

PMN can have considerable heterogeneity and plasticity, with the potential to enhance or suppress antitumor immunity $(43,57)$. Coffelt et al. (58) showed that PMN suppressed CTL responses and that depletion of IL-17 or G-CSF abrogated the T cell-suppressive phenotype in a mammary tumor model. PMN have also enhanced mammary tumor metastasis to lungs (59). Targeting CXCR2, which mediates PMN recruitment, suppressed tumorigenesis and metastasis in mice (60), and dual targeting of CXCR2 and CCR2 enhanced responses to chemotherapy (61). Activated PMN can also kill tumor cells (62). Eruslanov et al. (63) showed that TAN from early-stage lung cancer enhanced T cell responses. By contrast, our results show that PMN acquired a suppressor phenotype once exposed to the TME. Thus, the programming of PMN to a pro- or antitumorigenic phenotype depends on cues within the TME that include tumor-derived factors and products of inflammation and injury.

In response to infection, activation of CR3 on PMN leads to a signaling cascade that results in the phagocytosis of extracellular pathogens and downstream activation of Syk (64) and NADPH oxidase required for clearance of pathogens $(65,66)$. In the TME, our results point to several PMN effector functions that normally function to eradicate pathogens becoming barriers to antitumor immunity by impairing the activation and expansion of $\mathrm{T}$ cells. There is precedent for MDSC limiting $\mathrm{T}$ cell activation through ROS cross-signaling and inhibition of TCR signaling $(67,68)$. Studies are in progress to test whether NADPH oxidase-deficient PMN from CGD patients acquire a suppressor phenotype following ascites exposure. While a mechanism for PMN-mediated suppression is induction of $\mathrm{CD} 8^{+} \mathrm{T}$ cell apoptosis (69), we did not observe a clear effect of PMN and/or ascites on T cell viability. In addition, T cell proliferation was restored with ascites removal and restimulation, highlighting the potential for therapeutically reversing the quiescent phenotype of T cells. Gao et al. (70) described apoptotic PMN that suppress $\mathrm{T}$ cells in a cell contact- and ROS-dependent mechanism. While C3 inhibition with Cp40 fully abrogated the PMN suppressor phenotype, it did not significantly affect PMN viability. However, this finding doesn't exclude the potential for apoptosis/antiapoptosis pathways influencing the PMN suppressor phenotype.

Ascites induced the synthesis of a strikingly large number of proteins mediating multiple effector functions in PMN, which was required for the suppressor phenotype. Grassi et al. (71) identified stage-specific transcriptome and epigenetic changes in PMN maturation in which granular constituents are produced during granulopoiesis in the marrow and the signaling components that activate them are transcribed at later stages in circulation. Mature PMN maintain activated levels of PU.1 and can synthesize new proteins through a number of pathways (72-74). A limitation of this proteomics analysis is that we cannot discriminate between newly synthesized proteins versus intracellular transport of proteins from the ascites. Future studies will delineate the transcriptional and epigenetic changes in PMN induced by ascites exposure and the relationship with proteomic changes. 
Finally, we observed that postoperative drainage fluid induced the PMN suppressor phenotype similar to paired preoperative ascites. This finding was observed in patients with $\mathrm{R} 0$ and optimal debulking surgeries. Since primary surgery for advanced EOC is noncurative, postsurgical immunosuppression is likely to be clinically relevant. We observed that the new microenvironment was characterized by an early neutrophilic peritonitis, which others have reported should be followed by wound healing responses, including the accumulation of immunosuppressive cells that can promote growth of residual tumor (75). The concept of the protumorigenic effect of surgery is indirectly supported by short delays in adjuvant chemotherapy after primary surgery for EOC that correlate with shorter PFS and OS $(76,77)$. Our finding that postoperative drainage fluid induced the PMN suppressor phenotype is consistent with the findings that acute inflammatory conditions unrelated to cancer (e.g., LPS challenge, severe injury, and sepsis) can induce an expansion of suppressive granulocytes in humans $(31,78)$. These findings suggest that the expansion of suppressive PMN, both immature and mature, can occur in response to multiple insults and through distinct signaling pathways. Further studies are warranted to compare the transcriptional and proteomic profiles and functional characteristics of the PMN suppressor phenotype induced in the TME compared with other acute and chronic inflammatory conditions. The induction of suppressive PMN may be a strategy to limit tissue injury or avert autoimmunity by restraining $\mathrm{T}$ cell responses; however, in the TME, these same pathways are predicted to impede antitumor immunity.

\section{Methods}

Supplemental Methods are provided online with this article.

Patients and specimens. Participants included healthy donors, control female patients with a benign adnexal mass undergoing resection surgery, and cancer patients with malignant effusions. Healthy donors $(n=4)$ were White, aged 26-51, and equally divided between sexes. From 2015-2017, blood and ascites were collected from patients with newly diagnosed advanced (stage III or IV) ovarian cancer, as previously described (79). Blood was collected prior to primary surgery, and ascites were collected either by diagnostic paracentesis or in the operating room prior to surgery. Ascites were filtered through $300-\mu \mathrm{M}$ filters and then centrifuged $\left(500 \mathrm{~g}, 10\right.$ minutes). Aliquots of supernatants were stored at $-80^{\circ} \mathrm{C}$ until further use. When available, postoperative drainage fluid from an abdominal drainage tube was collected the day after primary surgery. Patients with early stage (I or II) or unstaged disease were excluded from the analysis. The medical records of these patients were retrospectively reviewed for demographics, tumor stage and grade, baseline serum CA125 levels, debulking status, and chemotherapy response. In 2018, malignant pleural effusions were collected by thoracentesis from patients with various metastatic cancers and processed following the same protocol.

Analysis of immune infiltrate in peripheral blood and ascites. Peripheral blood was collected in EDTA-coated tubes (Vacutainer, BD Biosciences). Whole blood was washed with PBS and centrifuged (500 g, 10 minutes). Cells from blood and ascites were analyzed by flow cytometry within 24 hours. Flow cytometry analysis was conducted on a Fortessa (Becton Dickinson). Forward scatter versus side scatter gating was set to include all nonaggregated cells from at least 20,000 events collected per sample. Data were analyzed using WinList 9.0.

Isolation of PMN and T cells from peripheral blood. PMN and T cells were isolated from peripheral blood $<1$ hour after collection using the MACSxpress Neutrophil Isolation Kit and the CD4, CD8, or Pan T cell Isolation Kits, respectively (Miltenyi Biotec). The purity of PMN was $>96 \%$ based on cytology and $\mathrm{CD} 45^{+} \mathrm{CD} 33^{\text {mid }} \mathrm{CD} 15^{+} \mathrm{CD} 6 \mathrm{~b}^{+}(80)$; there was complete concordance between $\mathrm{CD} 15$ and CD66b expression. The purity of $\mathrm{T}$ cells was $>97 \%$ based on $\mathrm{CD} 45^{+} \mathrm{CD} 3^{+}, \mathrm{CD} 45^{+} \mathrm{CD} 3^{+} \mathrm{CD} 4^{+}$, and $\mathrm{CD} 45^{+} \mathrm{CD}^{+} \mathrm{CD}^{+}$expression.

Statistics. All statistical analyses were performed using the R 3.4.0 statistical computing language. A nominal significance threshold of 0.05 was used unless otherwise specified. Statistical testing utilized 1-way ANOVA to determine significance, followed by a Tukey multiple comparisons post hoc test to determine which groups were significant. Prespecified interactions were tested within the ANOVA framework. The multivariate analysis comprised International Federation of Gynecology and Obstetrics (FIGO) stage, categorized as early (I, II, or IIIA/B) or late (IIIC or IV); histological grade; serum CA125 levels; debulking status (R0, defined as no macroscopic residual disease; optimal, defined by remaining disease $0.1-1 \mathrm{~cm}$; suboptimal, defined by remaining visible disease $>1 \mathrm{~cm}$ ); and platinum-sensitive versus refractory disease. 
Study approval. This study was approved by the IRB of Roswell Park Comprehensive Cancer Center (Buffalo, New York, USA) and was in compliance with federal and state requirements. All participants gave informed consent prior to inclusion in the study (protocols i215512 and i188310). All studies were conducted in compliance with the Declaration of Helsinki.

\section{Author contributions}

KLS and BHS developed the study and wrote the manuscript. KLS, TRE, ANHK, PCM, SS, JTW, J. Mark, and J. Matsuzaki designed and performed experiments and analyzed data. KM and KHE analyzed and described the statistical models and contributed to the manuscript. RCK, AMB, and KRM provided reagents. JTW, RBB, RCK, AMB, KRM, SR, JQ, KBM, SIA, KO, EZ, and BHS provided technical support and conceptual advice. All authors discussed the results and implications and commented on the manuscript at all stages.

\section{Acknowledgments}

This work was supported by Roswell Park Comprehensive Cancer Center grants NCI P30CA016056, the Roswell Park-UPCI Ovarian Cancer SPORE P50CA159981 (KO), 5R01CA188900 (BHS and KBM), R01CA172105 (SIA), 5T32CA108456 (PCM and JM), T32CA085183 (KLS), and K01LM012100 (KHE). The funders had no role in study design, data collection and analysis, decision to publish, or preparation of the manuscript. We would like to thank John D. Lambris (Department of Pathology and Laboratory Medicine, University of Pennsylvania Perelman School of Medicine, Philadelphia, Pennsylvania, USA) and William M. Nauseef (Department of Internal Medicine and Inflammation Program, Carver College of Medicine University of Iowa, Iowa City, Iowa, USA) for providing reagents and helpful discussions.

Address correspondence to: Brahm H. Segal, Department of Internal Medicine, Chief, Division of Infectious Diseases, Member, Department of Immunology, Roswell Park Comprehensive Cancer Center, Elm \& Carlton Streets, Buffalo, New York 14263, USA. Phone: 716.845.5721; Email: brahm.segal@roswellpark.org.

1. Jiménez-Sánchez A, et al. Heterogeneous Tumor-Immune Microenvironments among Differentially Growing Metastases in an Ovarian Cancer Patient. Cell. 2017;170(5):927-938.e20.

2. Worzfeld T, et al. The Unique Molecular and Cellular Microenvironment of Ovarian Cancer. Front Oncol. $2017 ; 7: 24$.

3. Idorn M, et al. Improved migration of tumor ascites lymphocytes to ovarian cancer microenvironment by CXCR2 transduction. Oncoimmunology. 2018;7(4):e1412029.

4. Obermajer N, Muthuswamy R, Odunsi K, Edwards RP, Kalinski P. PGE(2)-induced CXCL12 production and CXCR4 expression controls the accumulation of human MDSCs in ovarian cancer environment. Cancer Res. 2011;71(24):7463-7470.

5. Khan AN, et al. Targeting myeloid cells in the tumor microenvironment enhances vaccine efficacy in murine epithelial ovarian cancer. Oncotarget. 2015;6(13):11310-11326.

6. Shenoy GN, et al. Exosomes Associated with Human Ovarian Tumors Harbor a Reversible Checkpoint of T-cell Responses. Cancer Immunol Res. 2018;6(2):236-247.

7. Shender VO, et al. Proteome-metabolome profiling of ovarian cancer ascites reveals novel components involved in intercellular communication. Mol Cell Proteomics. 2014;13(12):3558-3571.

8. Biskup K, Braicu EI, Sehouli J, Tauber R, Blanchard V. The ascites N-glycome of epithelial ovarian cancer patients. J Proteomics. 2017; 157:33-39.

9. Worzfeld T, et al. Proteotranscriptomics Reveal Signaling Networks in the Ovarian Cancer Microenvironment. Mol Cell Proteomics. 2018;17(2):270-289.

10. Huang H, et al. Clinical significance of ascites in epithelial ovarian cancer. Neoplasma. 2013;60(5):546-552.

11. Szender JB, et al. Impact of ascites volume on clinical outcomes in ovarian cancer: A cohort study. Gynecol Oncol. 2017;146(3):491-497.

12. Zhang L, et al. Intratumoral T cells, recurrence, and survival in epithelial ovarian cancer. N Engl J Med. 2003;348(3):203-213.

13. Sato E, et al. Intraepithelial CD8+ tumor-infiltrating lymphocytes and a high CD8+/regulatory T cell ratio are associated with favorable prognosis in ovarian cancer. Proc Natl Acad Sci USA. 2005;102(51):18538-18543.

14. Curiel TJ, et al. Specific recruitment of regulatory $\mathrm{T}$ cells in ovarian carcinoma fosters immune privilege and predicts reduced survival. Nat Med. 2004;10(9):942-949.

15. Matsuzaki J, et al. Tumor-infiltrating NY-ESO-1-specific CD8+ T cells are negatively regulated by LAG-3 and PD-1 in human ovarian cancer. Proc Natl Acad Sci USA. 2010;107(17):7875-7880.

16. Brahmer JR, et al. Safety and activity of anti-PD-L1 antibody in patients with advanced cancer. N Engl JMed. 2012;366(26):2455-2465.

17. Hamanishi J, et al. Safety and Antitumor Activity of Anti-PD-1 Antibody, Nivolumab, in Patients With Platinum-Resistant Ovarian Cancer. J Clin Oncol. 2015;33(34):4015-4022. 
18. Matulonis UA, et al. Antitumor activity and safety of pembrolizumab in patients with advanced recurrent ovarian cancer: Interim results from the phase 2 KEYNOTE-100 study. J Clin Oncol. 2018;36(15 suppl):5511.

19. Veglia F, Perego M, Gabrilovich D. Myeloid-derived suppressor cells coming of age. Nat Immunol. 2018;19(2):108-119.

20. Bronte V, et al. Recommendations for myeloid-derived suppressor cell nomenclature and characterization standards. Nat Commun. 2016;7:12150.

21. Wu WC, et al. Circulating hematopoietic stem and progenitor cells are myeloid-biased in cancer patients. Proc Natl Acad Sci USA. 2014;111(11):4221-4226.

22. Netherby CS, et al. The Granulocyte Progenitor Stage Is a Key Target of IRF8-Mediated Regulation of Myeloid-Derived Suppressor Cell Production. J Immunol. 2017;198(10):4129-4139.

23. Cui TX, et al. Myeloid-derived suppressor cells enhance stemness of cancer cells by inducing microRNA101 and suppressing the corepressor CtBP2. Immunity. 2013;39(3):611-621.

24. Shaul ME, et al. Tumor-associated neutrophils display a distinct N1 profile following TGF $\beta$ modulation: A transcriptomics analysis of pro- vs. antitumor TANs. Oncoimmunology. 2016;5(11):e1232221.

25. Banerjee S, et al. A multicenter, randomized trial of flat dosing versus intrapatient dose escalation of single-agent carboplatin as first-line chemotherapy for advanced ovarian cancer: an SGCTG (SCOTROC 4) and ANZGOG study on behalf of GCIG. Ann Oncol. 2013;24(3):679-687.

26. Huang QT, et al. Prognostic Significance of Neutrophil-to-Lymphocyte Ratio in Ovarian Cancer: A Systematic Review and Meta-Analysis of Observational Studies. Cell Physiol Biochem. 2017;41(6):2411-2418.

27. Schmielau J, Finn OJ. Activated granulocytes and granulocyte-derived hydrogen peroxide are the underlying mechanism of suppression of t-cell function in advanced cancer patients. Cancer Res. 2001;61(12):4756-4760.

28. Rotondo R, et al. Exocytosis of azurophil and arginase 1-containing granules by activated polymorphonuclear neutrophils is required to inhibit T lymphocyte proliferation. J Leukoc Biol. 2011;89(5):721-727.

29. Tak T, et al. Neutrophil-mediated Suppression of Influenza-induced Pathology Requires CD11b/CD18 (MAC-1). Am J Respir Cell Mol Biol. 2018;58(4):492-499.

30. Singel KL, et al. Mitochondrial DNA in the tumour microenvironment activates neutrophils and is associated with worse outcomes in patients with advanced epithelial ovarian cancer. Br J Cancer. 2019;120(2):207-217.

31. Pillay J, et al. A subset of neutrophils in human systemic inflammation inhibits T cell responses through Mac-1. J Clin Invest. 2012;122(1):327-336.

32. Mastellos DC, et al. Compstatin: a C3-targeted complement inhibitor reaching its prime for bedside intervention. Eur J Clin Invest. 2015;45(4):423-440.

33. Lindorfer MA, et al. Compstatin Cp40 blocks hematin-mediated deposition of C3b fragments on erythrocytes: Implications for treatment of malarial anemia. Clin Immunol. 2016;171:32-35.

34. Nunn MA, et al. Complement inhibitor of C5 activation from the soft tick Ornithodoros moubata. J Immunol. 2005;174(4):2084-2091.

35. Blom AM, et al. A novel method for direct measurement of complement convertases activity in human serum. Clin Exp Immunol. 2014;178(1):142-153.

36. Uriarte SM, et al. Granule exocytosis contributes to priming and activation of the human neutrophil respiratory burst. J Immunol. 2011;187(1):391-400.

37. McLeish KR, Uriarte SM, Tandon S, Creed TM, Le J, Ward RA. Exocytosis of neutrophil granule subsets and activation of prolyl isomerase 1 are required for respiratory burst priming. J Innate Immun. 2013;5(3):277-289.

38. Lee W, Ko SY, Mohamed MS, Kenny HA, Lengyel E, Naora H. Neutrophils facilitate ovarian cancer premetastatic niche formation in the omentum. J Exp Med. 2019;216(1):176-194.

39. Wong JL, Obermajer N, Odunsi K, Edwards RP, Kalinski P. Synergistic COX2 Induction by IFN $\gamma$ and TNF $\alpha$ Self-Limits Type1 Immunity in the Human Tumor Microenvironment. Cancer Immunol Res. 2016;4(4):303-311.

40. Yang L, Zhang X, Ma Y, Zhao X, Li B, Wang H. Ascites promotes cell migration through the repression of miR-125b in ovarian cancer. Oncotarget. 2017;8(31):51008-51015.

41. McLane LM, et al. Differential localization of T-bet and Eomes in CD8 T cell memory populations. J Immunol. 2013;190(7):3207-3215.

42. Matsuzaki J, et al. Direct tumor recognition by a human CD4(+) T-cell subset potently mediates tumor growth inhibition and orchestrates anti-tumor immune responses. Sci Rep. 2015;5:14896.

43. Sagiv JY, et al. Phenotypic diversity and plasticity in circulating neutrophil subpopulations in cancer. Cell Rep. 2015;10(4):562-573.

44. Reis ES, Mastellos DC, Ricklin D, Mantovani A, Lambris JD. Complement in cancer: untangling an intricate relationship. Nat Rev Immunol. 2018;18(1):5-18.

45. Platt JL, et al. C3d regulates immune checkpoint blockade and enhances antitumor immunity. JCI Insight. 2017;2(9):e90201

46. Facciabene A, et al. Local endothelial complement activation reverses endothelial quiescence, enabling t-cell homing, and tumor control during t-cell immunotherapy. Oncoimmunology. 2017;6(9):e1326442.

47. Cho MS, et al. Autocrine effects of tumor-derived complement. Cell Rep. 2014;6(6):1085-1095.

48. Cho MS, et al. Complement Component 3 Is Regulated by TWIST1 and Mediates Epithelial-Mesenchymal Transition. J Immunol. 2016;196(3):1412-1418.

49. Yuen J, et al. NETosing Neutrophils Activate Complement Both on Their Own NETs and Bacteria via Alternative and Non-alternative Pathways. Front Immunol. 2016;7:137.

50. Blatt AZ, et al. Factor H C-Terminal Domains Are Critical for Regulation of Platelet/Granulocyte Aggregate Formation. Front Immunol. 2017;8:1586.

51. Liu CF, et al. Complement Receptor 3 Has Negative Impact on Tumor Surveillance through Suppression of Natural Killer Cell Function. Front Immunol. 2017;8:1602.

52. Medler TR, et al. Complement C5a Fosters Squamous Carcinogenesis and Limits T Cell Response to Chemotherapy. Cancer Cell. 2018;34(4):561-578.e6.

53. Ajona D, et al. A Combined PD-1/C5a Blockade Synergistically Protects against Lung Cancer Growth and Metastasis. Cancer 
Discov. 2017;7(7):694-703.

54. Nunez-Cruz S, et al. Genetic and pharmacologic inhibition of complement impairs endothelial cell function and ablates ovarian cancer neovascularization. Neoplasia. 2012;14(11):994-1004.

55. Lizcano A, et al. Erythrocyte sialoglycoproteins engage Siglec-9 on neutrophils to suppress activation. Blood. 2017;129(23):3100-3110.

56. Negorev D, et al. Human neutrophils can mimic myeloid-derived suppressor cells (PMN-MDSC) and suppress microbead or lectin-induced T cell proliferation through artefactual mechanisms. Sci Rep. 2018;8(1):3135.

57. Singel KL, Segal BH. Neutrophils in the tumor microenvironment: trying to heal the wound that cannot heal. Immunol Rev. 2016;273(1):329-343.

58. Coffelt SB, et al. IL-17-producing $\gamma \delta \mathrm{T}$ cells and neutrophils conspire to promote breast cancer metastasis. Nature. 2015;522(7556):345-348.

59. Wculek SK, Malanchi I. Neutrophils support lung colonization of metastasis-initiating breast cancer cells. Nature. 2015;528(7582):413-417.

60. Steele CW, et al. CXCR2 Inhibition Profoundly Suppresses Metastases and Augments Immunotherapy in Pancreatic Ductal Adenocarcinoma. Cancer Cell. 2016;29(6):832-845.

61. Nywening TM, et al. Targeting both tumour-associated CXCR2+ neutrophils and CCR2+ macrophages disrupts myeloid recruitment and improves chemotherapeutic responses in pancreatic ductal adenocarcinoma. Gut. 2018;67(6):1112-1123.

62. Clark RA, Klebanoff SJ. Neutrophil-mediated tumor cell cytotoxicity: role of the peroxidase system. J Exp Med. 1975;141(6):1442-1447.

63. Eruslanov EB, et al. Tumor-associated neutrophils stimulate T cell responses in early-stage human lung cancer. J Clin Invest. 2014;124(12):5466-5480.

64. Futosi K, Mócsai A. Tyrosine kinase signaling pathways in neutrophils. Immunol Rev. 2016;273(1):121-139.

65. Löfgren R, Serrander L, Forsberg M, Wilsson A, Wasteson A, Stendahl O. CR3, FcgammaRIIA and FcgammaRIIIB induce activation of the respiratory burst in human neutrophils: the role of intracellular $\mathrm{Ca}(2+)$, phospholipase $\mathrm{D}$ and tyrosine phosphorylation. Biochim Biophys Acta. 1999;1452(1):46-59.

66. Forsberg M, Löfgren R, Zheng L, Stendahl O. Tumour necrosis factor-alpha potentiates CR3-induced respiratory burst by activating p38 MAP kinase in human neutrophils. Immunology. 2001;103(4):465-472.

67. Nagaraj S, et al. Altered recognition of antigen is a mechanism of CD8+ T cell tolerance in cancer. Nat Med. 2007;13(7):828-835

68. Corzo CA, et al. Mechanism regulating reactive oxygen species in tumor-induced myeloid-derived suppressor cells. J Immunol. 2009;182(9):5693-5701.

69. Michaeli J, et al. Tumor-associated neutrophils induce apoptosis of non-activated CD8 T-cells in a TNF $\alpha$ and NO-dependent mechanism, promoting a tumor-supportive environment. Oncoimmunology. 2017;6(11):e1356965

70. Gao R, et al. Tumor cell-released autophagosomes (TRAP) enhance apoptosis and immunosuppressive functions of neutrophils. Oncoimmunology. 2018;7(6):e1438108.

71. Grassi L, et al. Dynamics of Transcription Regulation in Human Bone Marrow Myeloid Differentiation to Mature Blood Neutrophils. Cell Rep. 2018;24(10):2784-2794.

72. Bjerregaard MD, Jurlander J, Klausen P, Borregaard N, Cowland JB. The in vivo profile of transcription factors during neutrophil differentiation in human bone marrow. Blood. 2003;101(11):4322-4332.

73. Lindemann SW, Yost CC, Denis MM, McIntyre TM, Weyrich AS, Zimmerman GA. Neutrophils alter the inflammatory milieu by signal-dependent translation of constitutive messenger RNAs. Proc Natl Acad Sci USA. 2004;101(18):7076-7081.

74. Federzoni EA, et al. PU.1 is linking the glycolytic enzyme HK3 in neutrophil differentiation and survival of APL cells. Blood. 2012;119(21):4963-4970.

75. Predina J, et al. Changes in the local tumor microenvironment in recurrent cancers may explain the failure of vaccines after surgery. Proc Natl Acad Sci USA. 2013;110(5):E415-E424

76. Joseph N, et al. Delay in chemotherapy administration impacts survival in elderly patients with epithelial ovarian cancer. Gynecol Oncol. 2015;137(3):401-405.

77. Singh S, Guetzko M, Resnick K. Preoperative predictors of delay in initiation of adjuvant chemotherapy in patients undergoing primary debulking surgery for ovarian cancer. Gynecol Oncol. 2016;143(2):241-245.

78. Janols $\mathrm{H}$, et al. A high frequency of MDSCs in sepsis patients, with the granulocytic subtype dominating in gram-positive cases. J Leukoc Biol. 2014;96(5):685-693.

79. Kolomeyevskaya N, et al. Cytokine profiling of ascites at primary surgery identifies an interaction of tumor necrosis factor- $\alpha$ and interleukin-6 in predicting reduced progression-free survival in epithelial ovarian cancer. Gynecol Oncol. 2015;138(2):352-357.

80. Gustafson MP, et al. A method for identification and analysis of non-overlapping myeloid immunophenotypes in humans. PLOS ONE. 2015;10(3):e0121546. 